R. MIYAOKA

KODAI MATH. J.

14 (1991), 13-41

\title{
LIE CONTACT STRUCTURES AND NORMAL CARTAN CONNECTIONS
}

\author{
Dedicated to Professor Noboru Tanaka on his 60th birthday
}

\author{
By REIKo MiYAOKA
}

\section{§ 0. Introduction.}

Studies of $G$-structures and connections concerned with differential systems on a manifold have been deeply developed by $\mathrm{N}$. Tanaka in these ten years $[\mathrm{T} 1,2,3, \cdots]$.

Classical examples of $G$-structures are the projective and the conformal structures on a differentiable manifold. The former is a geometry with model space $P^{n}(\boldsymbol{R})$ and group $P L(n, \boldsymbol{R}),[\mathrm{KN}]$, the latter is with model space $S^{n}$, the Möbius space, and group $P O(n+1,1)$, [O].

Here, we study a Lie contact structure considered by H. Sato [S, SY], which is a geometry over $(2 n-1)$-dimensional contact manifolds with model space $T_{1} S^{n}$, the unit tangent bundle of the $n$-dimensional standard sphere, and group $P O(n+1,2)$. Since the grading of the Lie algebra of $O(n+1,2)$ is from -2 to 2 (of the second kind), the structure is much more complicated than the classical ones.

In this paper, we give basic facts on Lie contact structures in $\S 1$. In $\S 2$, we discuss on some examples. In particular, the structure given on the unit tangent bundle $T_{1} M$ of a riemannian manifold $M$ is important because it is related with both conformal structure of $M$ and contact structure of $T_{1} M$ [M2]. To investigate the relation, we must calculate the curvature of the normal Cartan connection defined in $\S 3$. In fact, by the connection theory due to $\mathrm{N}$. Tanaka $[T 1,2,3]$, which is thoroughly applicable to Lie contact structures, the normal Cartan connection determines the structure completely $(\S 4)$. Nevertheless, the concrete description of its torsion and curvature have not yet been done. In $\S 5$, we give an explicit description of these objects, proving at the same time the existence of the connection in a constructive way. All of these results are used in [M2] to calculate the curvature of the normal Cartan connection of the Lie contact structure on $T_{1} M$. Note that the definition of normal Cartan connections in [T2] is different from the one in [T3], the latter would be preferable theoretically. We adopt here the definition in [T3].

Received April 19, 1989; Revised April 26, 1990. 
Except for elementary facts, the argument in this paper is self-contained; the theory itself depends on [T2]. A resume of the present paper and [M2] is given in [M3]. The author would like to thank to Professors H. Sato, K. Yamaguchi, S. Tanno and K. Sakamoto for their valuable suggestions.

\section{$\S 1$. Preliminaries.}

Let $\boldsymbol{R}_{2}^{n+3}=\left\{x=\left(x^{0}, \cdots x^{n+2}\right), x^{2} \in \boldsymbol{R}\right\}$ be an $(n+3)$-dimensional real vector space endowed with a scalar product $\langle$,$\rangle with signature (+, \cdots,+,-,-)$ and let $\boldsymbol{R}_{1}^{n+2}=\left\{x \in \boldsymbol{R}_{2}^{n+3}, x^{n+2}=0\right\}$. Denote by $P^{n+2}$ and $P^{n+1}$ the associated projective spaces. Furthermore, let $\boldsymbol{R}^{n+1}=\left\{x \in \boldsymbol{R}_{1}^{n+2}, x^{n+1}=0\right\}$. By $\langle$,$\rangle , we denote the$ induced scalar product on $\boldsymbol{R}_{1}^{n+2}$ or on $\boldsymbol{R}^{n+1}$. Now, the unit sphere $S^{n}=$ $\left\{x \in \boldsymbol{R}^{n+1} \mid\langle x, x\rangle=1\right\}$ is naturally embedded in $P^{n+1}$ as a Möbius space $Q^{n}$,

$$
S^{n} \simeq Q^{n}=\left\{[y] \in P^{n+1} \mid\langle y, y\rangle=0\right\},
$$

by $x \rightarrow(x, 1) \in \boldsymbol{R}_{1}^{n+2}$. On the other hand, let $\Sigma$ be the set of all oriented hyperspheres in $S^{n} ; \Sigma=\left\{(m, \theta) \in S^{n} \times[0, \pi) \mid\right.$ an oriented hypersphere with center $m$ and radius $\theta\}$. Then $\Sigma$ is naturally embedded in $P^{n+2}$ as a quadratic $Q^{n+1}$,

$$
\Sigma \simeq Q^{n+1}=\left\{[k] \in P^{n+2} \mid\langle k, k\rangle=0\right\},
$$

by $(m, \theta) \rightarrow(m, \cos \theta, \sin \theta) \in \boldsymbol{R}_{2}^{n+3}$.

The Möbius group $L$ is, by definition, a group consists of projective transformations of $P^{n+1}$ preserving $Q^{n}$, and we have $L=P O(n+1,1)$. The Lie transformation group $G$ is, by definition, a group consists of projective transformations of $P^{n+2}$ preserving $Q^{n+1}$, and we get $G=P O(n+1,2)[P]$. Clearly we have $L \subset G$. Now, let $\Lambda^{2 n-1}=\left\{\right.$ lines in $Q^{n+1}$ generated by $\left(\left[k_{1}\right],\left[k_{2}\right]\right) \in Q^{n+1} \times Q^{n+1}$, $\left.\left\langle k_{1}, k_{2}\right\rangle=0\right\}$. Then we have

$$
T_{1} S^{n}=\left\{(u, v) \in S^{n} \times S^{n} \mid\langle u, v\rangle=0\right\} \simeq \Lambda^{2 n-1}
$$

under a mapping $(u, v) \rightarrow\left(\left[k_{1}\right],\left[k_{2}\right]\right)$, where $k_{1}=(u, 1,0), k_{2}=(v, 0,1)$. Since $G$ preserves $\langle$,$\rangle , it induces an action on \Lambda^{2 n-1}$. It is easy to see that $G$ acts on $\Lambda^{2 n-1}$ transitively and $\Lambda^{2 n-1}=G / G^{\prime}$ for an isotropy subgroup $G^{\prime}$ of $G$.

Choosing a suitable base of $\boldsymbol{R}_{2}^{n+3}$ so that $\langle u, v\rangle={ }^{t} u \varepsilon v$, where

$$
\varepsilon=\left(\varepsilon_{\alpha \beta}\right)=\left(\begin{array}{rcc}
0 & 0 & -I_{2} \\
0 & I_{n-1} & 0 \\
-I_{2} & 0 & 0
\end{array}\right) .
$$

we have $O(n+1,2)=\left\{\left.S \in G L(n+3, \boldsymbol{R})\right|^{t} S \varepsilon S=\varepsilon\right\}$ and $G=P O(n+1,2)$. The Lie algebra $g$ of $G$ is then given by $g=\left\{\left.X \in \mathfrak{g l}(n+3, \boldsymbol{R})\right|^{t} X \varepsilon+\varepsilon X=0\right\}$. Note that $g$ is a graded Lie algebra with grading $g=g_{-2}+g_{-1}+g_{0}+g_{1}+g_{2},\left[g_{\imath}, g_{\jmath}\right]=g_{2+\jmath}$, where 


$$
\begin{aligned}
& \mathfrak{g}_{-2}={ }^{t} \mathfrak{g}_{2}=\left\{\left(\begin{array}{lll}
0 & 0 & c \\
0 & 0 & 0 \\
0 & 0 & 0
\end{array}\right), p=\left(\begin{array}{rr}
0 & p \\
-p & 0
\end{array}\right)\right\}, \\
& \mathfrak{g}_{-1}={ }^{t} \mathfrak{g}_{1}=\left\{\left(\begin{array}{ccc}
0 & b & 0 \\
0 & 0 & { }^{t} b \\
0 & 0 & 0
\end{array}\right),{ }^{t} b \in \boldsymbol{R}^{n-1} \times \boldsymbol{R}^{n-1}\right\}, \\
& \mathfrak{g}_{0}=\left\{\left(\begin{array}{lll}
a & 0 & 0 \\
0 & e & 0 \\
0 & 0 & -{ }^{t} a
\end{array}\right), a \in \mathfrak{g l}(2, \boldsymbol{R}), e \in \mathfrak{D}(n-1)\right\} .
\end{aligned}
$$

LEMMA 1.2. The isotropy subgroup $G^{\prime}$ is given by

$$
G^{\prime}=\left\{\left(\begin{array}{ccc}
A & 0 & 0 \\
g d & g & 0 \\
A^{-1}\left(\frac{1}{2} t d d+f\right) & { }^{t} A^{-1 t} d & { }^{t} A^{-1}
\end{array}\right), \begin{array}{l}
g \in O(n-1), A \in G L(2, R) \\
d \in \boldsymbol{R}^{n-1} \times \boldsymbol{R}^{n-1}, f:=\left(\begin{array}{cc}
0 & -q \\
q & 0
\end{array}\right)
\end{array}\right\}
$$

The Lie algebra of $G^{\prime}$ is $\mathfrak{g}^{\prime}=g_{0}+g_{1}+g_{2}$, and $\operatorname{dim} G^{\prime}=\frac{1}{2}\left(n^{2}+n+8\right)$.

Proof. This follows from [T2, Lemma 2.5, 6]. In fact, we have

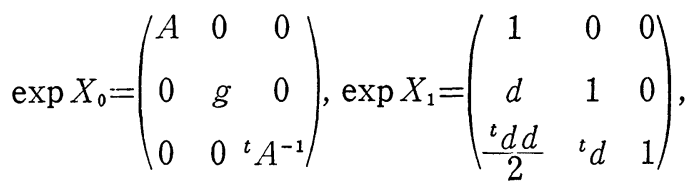

$$
\begin{aligned}
& \exp X_{2}=\left(\begin{array}{ccc}
1 & 0 & 0 \\
0 & 1 & 0 \\
f & 0 & 1
\end{array}\right), \quad X_{i} \in \mathfrak{g}_{\imath}, i=0,1,2 \text {, }
\end{aligned}
$$

where $A, g, d, f$ are given above.

q.e.d.

Now, we give an explicit description of the isotropy representation $\rho: G^{\prime} \rightarrow$ $G L(2 n-1)$. Note that $\mathfrak{g}=\mathfrak{g}^{\prime}+\mathfrak{m}$ where $\mathfrak{m}=T_{0}\left(G / G^{\prime}\right)$ We choose a base $e_{1}, e_{2}, \cdots, e_{2 n-1}$ of $\mathfrak{m}$ as follows

$$
e_{1}=\left(\begin{array}{lll}
0 & 0 & c \\
0 & 0 & 0 \\
0 & 0 & 0
\end{array}\right), \quad c=\left(\begin{array}{rr}
0 & 1 \\
-1 & 0
\end{array}\right)
$$




$$
\begin{aligned}
& e_{\imath}=\left(\begin{array}{ccc}
0 & b_{i} & 0 \\
0 & 0 & { }^{t} b_{i} \\
0 & 0 & 0
\end{array}\right), \quad b_{i}=\left(\begin{array}{l}
0, \cdots, 0, \stackrel{2}{1}, 0, \cdots, 0 \\
0, \ldots \ldots \ldots \ldots \ldots \ldots ., 0
\end{array}\right), \quad i=2, \cdots, n, \\
& e_{n+\imath-1}=\left(\begin{array}{ccc}
0 & b_{i}^{\prime} & 0 \\
0 & 0 & { }^{t} b_{i}^{\prime} \\
0 & 0 & 0
\end{array}\right), \quad b_{i}^{\prime}=\left(\begin{array}{l}
0, \cdots \cdots \cdots \cdots \cdots \cdots, 0 \\
0, \cdots, 0,1,0, \cdots, 0
\end{array}\right), \quad i=2, \cdots, n .
\end{aligned}
$$

PROPOSITION 1.3. With respect to this base, the isotropy representation $\tilde{G}=$ $\rho\left(G^{\prime}\right)$ is obtained by

$$
\tilde{G}=\left\{\left(\begin{array}{ccc}
s_{0}^{0} s_{1}^{1}-s_{1}^{0} s_{0}^{1} & 0 & 0 \\
-s_{0}^{0} s_{1}^{2}+s_{1}^{0} s_{0}^{2} & s_{0}^{0} s_{\jmath}^{2} & s_{1}^{0} s_{\jmath}^{2} \\
-s_{0}^{1} s_{1}^{2}+s_{1}^{1} s_{0}^{2} & s_{0}^{1} s_{\jmath}^{2} & s_{1}^{1} s_{j}^{2}
\end{array}\right),\left(s_{\beta}^{\alpha}\right) \in G^{\prime}, i, j=2, \cdots, n\right\} .
$$

Remark. With respect to a natural embedding $O(n-1) \subset C O(n-1) \subset G^{\prime}$, we have

$$
O(n-1) \subset C O(n-1) \subset \tilde{G}
$$

Proof. For $S=\left(s_{\beta}^{\alpha}\right) \in G^{\prime}$, we will calculate $S e_{I} S^{-1}$ modulo $g^{\prime}, I=1, \cdots, 2 n-1$. By Lemma 1.2, we have

so that

$$
S=\left(\begin{array}{ccc}
A & 0 & 0 \\
g d & g & 0 \\
{ }^{t} A^{-1}\left(\frac{1}{2} t d d+f\right) & { }^{t} A^{-1 t} d & { }^{t} A^{-1}
\end{array}\right),
$$

Then we have

$$
S^{-1}=\left(\begin{array}{ccc}
A^{-1} & 0 & 0 \\
-d A^{-1} & { }^{t} g & 0 \\
\left(\frac{1}{2}-t d-f\right) A^{-1} & -{ }^{t} d^{t} g & { }^{t} A
\end{array}\right) \text {. }
$$

$$
\begin{aligned}
S e_{1} S^{-1} & =\left(\begin{array}{ccc}
* & -A c^{t} d^{t} g & A c^{t} A \\
* & * & g d c^{t} A \\
* & * & *
\end{array}\right), \\
S e_{i} S^{-1} & =\left(\begin{array}{ccc}
* & A b_{i}{ }^{t} g & 0 \\
* & * & g^{t} b_{i}{ }^{t} A \\
* & * & *
\end{array}\right), \quad i=2, \cdots, n,
\end{aligned}
$$


and

Noting that

$$
S e_{n+\imath-1} S^{-1}=\left(\begin{array}{ccc}
* & A b_{i}^{\prime}{ }^{t} g & 0 \\
* & * & g^{t} b_{i}^{\prime} A \\
* & * & *
\end{array}\right), \quad i=2, \cdots, n .
$$

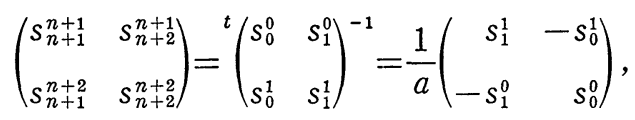

where

$$
a=s_{0}^{0} s_{1}^{1}-s_{1}^{0} s_{0}^{1},
$$

we get the proposition.

q.e.d.

DEFINITION. For a $(2 n-1)$-dimensional differentiable manifold $M$, a $\tilde{G}$ reduction of the linear frame bundle of $M$ is called a Lie frame bundle over $M$ or $a \tilde{G}$-structure on $M$.

Now, we define a Lie contact structure. For a $\tilde{G}$-structure $(P, \theta)$ on a $(2 n-1)$-dimensional manifold, the basic (usually, called a canonical) form $\theta$ is, by definition, an $\mathfrak{m}$-valued 1 -form on $P$ given by

$$
\theta\left(X_{u}\right)=u^{-1} \pi_{*} X, \quad X \in T_{u} P, \quad \pi: P \rightarrow M, \text { the projection. }
$$

DEFINITION. Let $(P, \theta)$ be a $\tilde{G}$-structure on a $(2 n-1)$-dimensional manifold $M$. Let $\theta_{p}$ be the $\mathfrak{g}_{p}$-component of $\theta$, where $\mathfrak{m}=\mathfrak{g}_{-2}+\mathfrak{g}_{-1}$. When $\theta$ satisfies

$$
d \theta_{-2}+\frac{1}{2}\left[\theta_{-1}, \theta_{-1}\right] \equiv 0 \quad\left(\bmod \theta_{-2}\right),
$$

the $\tilde{G}$-structure is called of type $\mathfrak{m}$, or equivalently, a Lie contact structure.

Clearly, a Lie contact structure is the $\tilde{G}$-structure of type $\mathfrak{m}$ discussed in $[\mathrm{T} 1,2,3]$ when $G=P O(n+1,2)$. It is mentioned by H. Sato [S] that there exists a Lie contact structure on the unit tangent bundle $T_{1} M$ of any $n$-dimensional Riemannian manifold. We show this in the next section. More generally, the structure possibly exists, on a $(2 n-1)$-dimensional contact manifold, as we investigate in $\S 2$.

\section{$\S 2$. The Lie contact structure.}

In $[T 1$, p. 10$]$, for a regular differential system $D$ on $M$, Tanaka constructed a graded Lie algebra $\mathfrak{m}(x)$ at each point $x$ of $M$, and when $\mathfrak{m}(x)$ is isomorphic to a fixed fundamental graded Lie algebra $\mathfrak{m}=\sum_{p<0} \mathrm{~g}_{p}$ for all $x$, he calls $D$ of type $\mathfrak{m}$. Moreover, he shows that if $(M, D)$ is a regular differential system of type $\mathfrak{m}$, then it corresponds to a $G_{0}(\mathfrak{m})^{\#}$-structure $\left(P^{\#}, \theta\right)$ of type $\mathfrak{m}$ over $M$, 


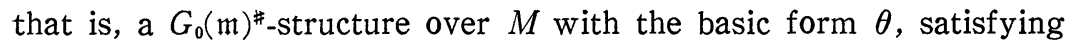

$$
d \theta_{p}+\frac{1}{2} \sum_{r+s=p}\left[\theta_{r}, \theta_{s}\right] \equiv 0 \quad\left(\bmod \theta_{r}(r \leq p) ; \theta_{r} \wedge \theta_{s}, r+s<p, p<r, s<0\right),
$$

where $p \leq-2$ and $\theta_{p}$ denotes the $g_{p}$-component of $\theta$. For the definition of $\left(P^{\#}, \theta\right)$ and $G_{0}(\mathfrak{m})^{\#}$, in general cases, see [T1]. This is a beautiful geometric interpretation of $(M, D)$, and the more important is the existence of a normal Cartan connection on $\left(P^{\#}, \theta\right)$ when the structure group satisfies a certain condition.

When $D$ is, in particular, a contact structure of a $(2 n-1)$-dimensional manifold $M,(2.1)$ is reduced to a simple equation (1.2). We will explain this case in detail. At each point $x$ of $M$, put $\mathfrak{g}_{-2}(x)=T_{x} M / D_{x}, \mathfrak{g}_{-1}(x)=D_{x}$ and $\mathfrak{m}(x)=$ $\mathfrak{g}_{-2}(x)+\mathfrak{g}_{-1}(x)$. A Lie bracket in $\mathfrak{m}(x)$ is defined by $\left[\mathfrak{m}(x), \mathfrak{g}_{-2}(x)\right]=0$, and for $X, Y \in \mathrm{g}_{-1}(x),[X, Y]=\pi([X, Y])$, where $\pi: T_{x} M \rightarrow \mathrm{g}_{-2}(x)$ is the projection. This is well-defined since $\pi([f X, Y])=f \pi([X, Y])$ for a function on $M$. We put a generic assumption that $\mathfrak{m}(x)$ is isomorphic to a fundamental graded Lie algebra $\mathfrak{m}$, at each $x \in M$. Now, let $P^{\#}$ be the set of frames on $M$ satisfying

(1) $z: \mathfrak{g}_{-1} \rightarrow g_{-1}(x)$ a linear isomorphism,

(2) $\tilde{z}: \mathfrak{m} \rightarrow \mathfrak{m}(x)$ a Lie algebra isomorphism,

where $\tilde{z}$ is a map naturally induced from $z: \mathfrak{m} \rightarrow T_{x} M$. Let $G_{0}(\mathfrak{m})$ be the automorphism group of $\mathfrak{m}, N_{0}=\left\{c \in G L(\mathfrak{m}) \mid c Y_{-2} \equiv Y_{-2}\left(\bmod \mathfrak{g}_{-1}\right), c Y_{-1}=Y_{-1}, Y_{p} \in \mathfrak{g}_{p}\right\}$ and $G_{0}(\mathfrak{m})^{\#}=G_{0}(\mathfrak{m}) \cdot N_{0}$. Actually, $P^{\#}$ is a $G_{0}(\mathfrak{m})^{\#}$-bundle over $M$.

LEMma 2.1. Let $\left(M^{2 n-1}, D\right)$ be a contact manifold and let $\left(P^{\#}, \theta\right)$ be the associated $G_{0}(\mathfrak{m})^{\#}$-structure with basic form $\theta$. Then it satisfies

$$
d \theta_{-2}+\frac{1}{2}\left[\theta_{-1}, \theta_{-1}\right] \equiv 0 \quad\left(\bmod \theta_{-2}\right) .
$$

Proof. As is well known [S2], with a contact manifold $M$ is associated a contact metric structure $(\eta, \varphi, \xi, g), \eta$ the contact form, $\varphi$ is an endomorphism of $T M, \xi$ is a vector field on $M$ and $g$ a metric on $M$ satisfying

$$
\begin{aligned}
& \eta(\xi)=1, \\
& \varphi^{2}=-i d+\eta \otimes \xi, \\
& g(\varphi X, \varphi Y)=g(X, Y)-\eta(X) \eta(Y), \quad X, Y \in T M, \\
& d \eta(X, Y)=g(X, \varphi Y), \quad X, Y \in T M .
\end{aligned}
$$

Let $\theta$ be the canonical form on $P^{\#}$. We can choose a local section $\sigma: M \rightarrow P^{\#}$, $\sigma(x)=\left(z_{1}, z_{2}, \cdots, z_{n}, z_{\overline{2}}, \cdots, z_{\bar{n}}\right)$ where $z_{1}=2 \xi, z_{2}$ is a unit vector orthogonal to $\xi, z_{\overline{2}}=\varphi z_{2}, z_{3}$ is a unit vector orthogonal to $z_{1}, z_{2}$ and $z_{\overline{2}}, \cdots$, and so forth. Note that $\varphi \xi=0$ and $\eta\left(z_{\imath}\right)=\eta\left(z_{i}\right)=0$ for $i=2, \cdots, n$ [B, p. 20]. Construct a Lie algebra $\mathfrak{m}$ consisting of a base $e_{1}, \cdots, e_{n}, e_{\overline{2}}, \cdots, e_{\bar{n}}$ where the Lie bracket is defined by

$$
\left[e_{1}, e_{\imath}\right]=\left[e_{1}, e_{i}\right]=\left[e_{\imath}, e_{j}\right]=\left[e_{i}, e_{j}\right]=0,\left[e_{\imath}, e_{j}\right]=\delta_{i j} e_{1} \text {. }
$$


Then from (2.3), we have

$$
d \eta\left(z_{\imath}, z_{j}\right)=-\frac{1}{2} \eta\left(\left[z_{\imath}, z_{j}\right]\right)=g\left(z_{\imath}, \varphi z_{j}\right)=-\delta_{i \jmath},
$$

and $d \eta\left(z_{\alpha}, z_{\beta}\right)=0$ for other pair $\left(z_{\alpha}, z_{\beta}\right)$, which implies that $D$ is of type $\mathfrak{m}$. Put $\bar{\theta}=\sigma^{*} \theta$. Since we have an isomorphism $\sigma(x): \mathfrak{m} \rightarrow T_{x} M, x \in M$, given by $\sigma(x)\left(a^{\beta} e_{\beta}\right)=a^{\beta} z_{\beta}$ for $a^{\beta} z_{\beta} \in T_{x} M$, where $\beta$ is summed over $1, \cdots, n, \overline{2}, \cdots, \bar{n}$, we see that

$$
\bar{\theta}\left(a^{\beta} z_{\beta}\right)=\theta\left(\sigma_{*} a^{\beta} z_{\beta}\right)=\sigma(x)^{-1} a^{\beta} z_{\beta}=a^{\beta} e_{\beta} .
$$

Thus we can write

$$
\bar{\theta}_{-2}=\frac{1}{2} \eta e_{1}, \quad \bar{\theta}_{-1}=\sum_{i=2}^{n}\left(\bar{\theta}^{i} e_{i}+\bar{\theta}^{\bar{i}} e_{i}\right) .
$$

Then from (2.3) and (2.4), we obtain

$$
d \bar{\theta}_{-2}+\frac{1}{2}\left[\bar{\theta}_{-1}, \bar{\theta}_{-1}\right]=0 .
$$

Now, at $u=\sigma(x) a^{-1} \in P^{\#}, a=b c, b \in G_{0}(\mathfrak{m}), c \in N_{0}$, identifying $\bar{\theta}=\pi * \bar{\theta}$, we have

i. e.

$$
\theta=a \bar{\theta}=b c \bar{\theta},
$$

$$
\theta_{-2}=b \bar{\theta}_{-2}, \quad \text { and } \quad \theta_{-1}=b \bar{\theta}_{-1}+b\left(c \bar{\theta}_{-2}\right)_{-1} \equiv b \bar{\theta}_{-1} \quad\left(\bmod \bar{\theta}_{-2}\right) .
$$

Therefore, we get

$$
d \theta_{-2} \equiv b d \bar{\theta}_{-2}\left(\bmod \bar{\theta}_{-2}\right)
$$

but since

$$
\bar{\theta}_{-2}=b^{-1} \theta_{-2},
$$

we have

$$
d \theta_{-2} \equiv b d \bar{\theta}_{-2} \quad\left(\bmod \theta_{-2}\right) .
$$

On the other hand, form $\bar{\theta}_{-1} \equiv b^{-1} \theta_{-1}\left(\bmod \bar{\theta}_{-2}\right),(2.6)$ and $(2.7)$, it follows

$$
d \bar{\theta}_{-2}=-\frac{1}{2}\left[\bar{\theta}_{-1}, \bar{\theta}_{-1}\right] \equiv-\frac{1}{2} b^{-1}\left[\theta_{-1}, \theta_{-1}\right]\left(\bmod \theta_{-2}\right),
$$

and we get the lemma.

q.e.d.

By using the base $\left(e_{1}, e_{2}, e_{i}\right)$ of $\mathfrak{m}$ above, the structure group $G_{0}(\mathfrak{m})^{\#}$ can be represented as

and so contains $\tilde{G}$.

$$
G_{0}(\mathfrak{m})^{\#}=\left\{\left(\begin{array}{cc}
a & 0 \\
* & C S P(n-1, \boldsymbol{R})
\end{array}\right), a \neq 0\right\},
$$


COROLlaRY 2.2. For a contact manifold $\left(M^{2 n-1}, D\right), a \tilde{G}$-reduction of $\left(P^{\#}, \theta\right)$ is a Lie contact structure.

Noting (1.1), we show :

Proposition 2.3. Let $(M, g)$ be an n-dimensional Riemannian manifold. Then on the unit tangent bundle $T_{1} M$ of $M$ exists a Lie contact structure.

Proof. Let $Q_{g}$ be the principal fiber bundle over $M$ with structure group $O(n)$. Then by [KNI, p. 57], $P_{g}=\left(Q_{g} / O(n-1), O(n-1)\right)$ is the principal fiber bundle over $T_{1} M$. Noting (1.1), we define an extended buncle

$$
\tilde{P}_{g}=P_{g} \times_{O(n-1)} \tilde{G} \text {. }
$$

In order to show that this $\tilde{G}$-structure is of type $\mathfrak{m}$, recall the geometry of $T_{1} M$ when $M$ is a riemannian manifold $[\mathrm{S} 1,3]$ : For $z \in Q_{g}$, where $z=\left(z_{1}, \cdots, z_{n}\right)$, let $z_{\imath}^{h}, z_{i}^{v} \in T T M$ be the horizontal and the vertical lift of each vector $z_{\imath}$. Then for $z_{1} \in T_{1} M, u(z)=\left(z_{1}^{h}, z_{\imath}^{h}, z_{\imath}^{v}\right), 2 \leq i \leq n$, is an orthonormal base of $T_{z_{1}} T_{1} M$ with respect to the induced Sasakian metric $S_{g}$ on $T_{1} M$. Thus $P_{g}=\left\{u(z) \mid z \subseteq Q_{g}\right\}$ is an $O(n-1)$-principal bundle over $T_{1} M$ under the action $\sigma$ of $h \in O(n-1)$ given by $\sigma(h) u(z)=u(z \tilde{h})$ where

$$
\tilde{h}=\left(\begin{array}{ll}
1 & 0 \\
0 & h
\end{array}\right) \in O(n) .
$$

Now, on the contact structure $\left(T_{1} M, D\right)$ defined by

$$
D=\left\{X \in T_{z_{1}} T_{1} M \mid S_{g}\left(z_{1}^{h}, X\right)=0\right\},
$$

the associated contact metric structure $(\eta, \xi, \varphi, \tilde{g})$ is given by

$$
\begin{aligned}
& \tilde{g}=\frac{1}{4} S_{g}, \quad \xi=2 z_{1}^{h}, \quad \eta \text { is the dual to } \xi w . r . t . \tilde{g} \\
& \varphi(\xi)=0, \quad \varphi\left(z_{\imath}^{h}\right)=z_{\imath}^{v}, \quad \varphi\left(z_{\imath}^{v}\right)=-z_{\imath}^{h}, \quad i=2, \cdots, n,
\end{aligned}
$$

[B, p. 133], using $u(z), z \in Q_{g}$. Thus (2.5) is satisfied for $z_{\imath}=2 z_{\imath}^{h}, z_{j}=2 z_{\jmath}^{v}$, $1 \leq i \leq n, 2 \leq j \leq n$, and hence we get $\left[z_{\imath}^{h}, z_{\imath}^{v}\right]=z_{1}^{h}$, which implies that $u(z)$ is a frame adapted to the structure. Thus the proposition follows from Corollary 2.2 .

q.e.d.

Remark 2.4. The Lie contact structure on $T_{1} M$ defined as above depends, in fact, only on the conformal structure of $M$ [see SY].

\section{§3. A normal Cartan connection of type $G / G^{\prime}$.}

To a Lie contact structure, we can apply the theory of N. Tanaka [T3] which guarantees the existence of a normal Cartan connection of type $G / G^{\prime}$. 
In $\S 5$, we prove this fact directly in a constructive way. This section is a preliminary for it.

We fix a base of $g=\sum_{p=-2}^{2} g_{p}$ as follows; let $e_{1}, e_{\imath}, e_{i}=e_{n+\imath-1}, 2 \leq i \leq n$, be the base of $\mathfrak{m}$ defined in $\S 1$. Define $e_{1}^{*}={ }^{t} e_{1}, e_{2}^{*}={ }^{t} e_{2}$ and $e_{i}^{*}={ }^{t} e_{i}(i=2, \cdots, n)$ which form a base of $\mathfrak{m}^{*}=\mathrm{g}_{1}+\mathrm{g}_{2}$. Furthermore, let $E_{\jmath}^{i}$ denote the $2 \times 2$ matrix with $(i, j)$-component $=1$ and the others zero. Define

$$
\begin{array}{ll}
e_{0}^{0}=\left(\begin{array}{llr}
E_{1}^{1} & 0 & 0 \\
0 & 0 & 0 \\
0 & 0 & -E_{1}^{1}
\end{array}\right), \quad e_{1}^{0}=\left(\begin{array}{llr}
E_{2}^{1} & 0 & 0 \\
0 & 0 & 0 \\
0 & 0 & -E_{1}^{2}
\end{array}\right), \quad e_{0}^{1}=\left(\begin{array}{llr}
E_{1}^{2} & 0 & 0 \\
0 & 0 & 0 \\
0 & 0-E_{2}^{1}
\end{array}\right), \\
e_{1}^{1}=\left(\begin{array}{llr}
E_{2}^{2} & 0 & 0 \\
0 & 0 & 0 \\
0 & 0 & -E_{2}^{2}
\end{array}\right), \quad e_{\jmath}^{2}=\left(\begin{array}{lll}
0 & 0 & 0 \\
0 & F_{\jmath}^{\imath} & 0 \\
0 & 0 & 0
\end{array}\right),
\end{array}
$$

where $F_{\jmath}^{i}, 2 \leq i<j \leq n$, is the $(n-1) \times(n-1)$ matrix with $(i, j)$-component $=-(j, i)$ component $=1$ and the others zero. Put $e_{j}^{2}=-e_{\imath}^{j}$ if $i>j$. The following is elementary.

LEMMA 3.1. The Lie bracket $[a, b]$ of $\mathrm{g}$ is given in this base by the follow-

\begin{tabular}{|c|c|c|c|c|c|c|c|c|c|c|c|}
\hline$a$ & $e_{1}$ & $e_{\jmath}$ & $e_{j}$ & $e_{0}^{0}$ & $e_{1}^{0}$ & $e_{0}^{\prime}$ & $e_{1}^{1}$ & $e_{\jmath}^{k}$ & $e_{J}^{*}$ & $e_{J}^{*}$ & $e_{1}^{*}$ \\
\hline$e_{1}$ & 0 & 0 & 0 & $-e_{1}$ & 0 & 0 & $-e_{1}$ & 0 & $-e_{j}$ & $e_{j}$ & $E$ \\
\hline$e_{\imath}$ & 0 & 0 & $\delta_{j}^{\imath} e_{1}$ & $-e_{\imath}$ & 0 & $-e_{\bar{\imath}}$ & 0 & $E_{\imath \jmath}{ }^{k}$ & $\delta_{j}^{i} e_{0}^{0}+e_{j}^{i}$ & $\delta_{j}^{i} e_{1}^{0}$ & $-e_{\imath}^{*}$ \\
\hline$e_{\bar{i}}$ & 0 & $-\delta_{j}^{2} e_{1}$ & 0 & 0 & $e_{\imath}$ & 0 & $-e_{i}$ & $E_{i j}^{k}$ & $\delta_{\jmath}^{2} e_{0}^{1}$ & $\delta_{j}^{2} e_{1}^{1}+e_{j}^{i}$ & $e_{i}^{*}$ \\
\hline$e_{0}^{0}$ & $e_{1}$ & $e_{j}$ & 0 & 0 & $e_{1}^{0}$ & $-e_{0}^{1}$ & 0 & 0 & $-e_{\jmath}^{*}$ & 0 & $-e_{1}^{*}$ \\
\hline$e_{1}^{0}$ & 0 & 0 & $-e$, & $-e_{1}^{0}$ & 0 & $F$ & $e_{1}^{0}$ & 0 & $-e_{j}^{*}$ & 0 & 0 \\
\hline$e_{0}^{1}$ & 0 & $e_{j}$ & 0 & $e_{0}^{1}$ & $-F$ & 0 & $-e_{0}^{1}$ & 0 & 0 & $-e_{\jmath}^{*}$ & 0 \\
\hline$e_{1}^{1}$ & $e_{1}$ & 0 & $e_{j}$ & 0 & $-e_{1}^{0}$ & $e_{0}^{1}$ & 0 & 0 & 0 & $-e_{j}^{*}$ & $-e_{1}^{*}$ \\
\hline$e_{m}^{2}$ & 0 & $-E_{\jmath m}^{2}$ & $-E_{j m}^{2}$ & 0 & 0 & 0 & 0 & $A$ & $-E_{\jmath m}^{* \imath}$ & $-E_{j m}^{* \imath}$ & 0 \\
\hline$e_{i}^{*}$ & $e_{i}$ & $e_{j}^{2}-\delta_{j}^{i} e_{0}^{0}$ & $-\delta_{j}^{i} e_{0}^{1}$ & $e_{i}^{*}$ & $e_{i}^{*}$ & 0 & 0 & $E_{\imath \jmath}^{* k}$ & 0 & $-\delta_{j}^{i} e_{1}^{*}$ & 0 \\
\hline$e_{i}^{*}$ & $-e_{\imath}$ & $-\delta_{j}^{2} e_{1}^{0}$ & $e_{j}^{i}-\delta_{j}^{i} e_{1}^{1}$ & 0 & 0 & $e_{\imath}^{*}$ & $e_{i}^{*}$ & $E_{i j}^{* k}$ & $\delta_{j}^{i} e_{1}^{*}$ & 0 & 0 \\
\hline$e_{1}^{*}$ & $-E$ & $e_{J}^{*}$ & $-e_{J}^{*}$ & $e_{1}^{*}$ & 0 & 0 & $e_{1}^{*}$ & 0 & 0 & 0 & 0 \\
\hline
\end{tabular}
ing table: 
where

$$
E=e_{0}^{0}+e_{1}^{1}, \quad F=e_{0}^{0}-e_{1}^{1}, \quad E_{i j}^{\varepsilon k}=e_{j}^{\varepsilon} \delta_{i}^{k}-e_{k}^{\varepsilon} \delta_{\jmath}^{i}, \quad E_{i j}^{\varepsilon k}=e_{j}^{\varepsilon} \delta_{i}^{k}-e_{k}^{\varepsilon} \delta_{j}^{2},
$$

$\varepsilon=$ nothing or $*, A=\delta_{j}^{i} e_{k}^{m}+\delta_{k}^{m} e_{\jmath}^{2}-\delta_{\jmath}^{m} e_{k}^{2}-\delta_{k}^{i} e_{\jmath}^{m}$.

From this table, we get

Lemma 3.2. The Killing form $B$ of $\mathfrak{g}$ gives the duality between $\mathfrak{m}$ and $\mathfrak{m}^{*}$ :

$$
\begin{aligned}
& B\left(e_{1}, e_{1}^{*}\right)=2(n+1), B\left(e_{\imath}, \mathrm{e}_{\jmath}^{*}\right)=B\left(e_{i}, e_{j}^{*}\right)=2(n+1) \delta_{i_{\jmath}}, \\
& \text { and } B(,)=0, \quad \text { for other pairs. }
\end{aligned}
$$

Now, we give the Mauer-Cartan equation of $G$, taking a local coordinate $\left(s_{n+2}^{0}, s_{n+1}^{2}, s_{n+2}^{2}, s_{0}^{0}, s_{1}^{0}, s_{0}^{1}, s_{1}^{1}, s_{\jmath}^{2}, s_{0}^{2}, s_{1}^{2}, s_{0}^{n+2}\right), 2 \leq i, j \leq n$, of $G$, where $\left(s_{\beta}^{\alpha}\right) \in G$.

LEMMA 3.3. Let $\omega_{\beta}^{\alpha}$ be the left invariant 1-form on $G$ which coincides with $d s_{\beta}^{\alpha}$ at $i d \in G$, where $(\alpha, \beta)$ moves so that $\left(s_{\beta}^{\alpha}\right)$ gives the local coordinate defined above. Then the Mauer-Cartan equation is given by

$$
\begin{aligned}
& d \omega_{n+2}^{0}=-\left(\omega_{0}^{0}+\omega_{1}^{1}\right) \wedge \omega_{n+2}^{0}-\omega_{i}^{0} \wedge \omega_{n+2}^{i}, \\
& d \omega_{n+1}^{2}=\omega_{1}^{2} \wedge \omega_{n+2}^{0}-\omega_{j}^{2} \wedge \omega_{n+1}^{3}+\omega_{n+1}^{2} \wedge \omega_{0}^{0}+\omega_{n+2}^{2} \wedge \omega_{1}^{0}, \\
& d \omega_{n+2}^{2}=-\omega_{0}^{2} \wedge \omega_{n+2}^{0}-\omega_{j}^{2} \wedge \omega_{n+2}^{3}+\omega_{n+1}^{2} \wedge \omega_{0}^{1}+\omega_{n+2}^{i} \wedge \omega_{1}^{1}, \\
& d \omega_{0}^{0}=-\omega_{1}^{0} \wedge \omega_{0}^{1}-\omega_{n+1}^{2} \wedge \omega_{0}^{2}-\omega_{n+2}^{0} \wedge \omega_{0}^{n+2}, \\
& d \omega_{1}^{0}=-\left(\omega_{0}^{0}-\omega_{1}^{1}\right) \wedge \omega_{1}^{0}-\omega_{n+1}^{2} \wedge \omega_{1}^{2}, \\
& d \omega_{0}^{1}=\left(\omega_{0}^{0}-\omega_{1}^{1}\right) \wedge \omega_{0}^{1}-\omega_{n+2}^{2} \wedge \omega_{0}^{2}, \\
& d \omega_{1}^{1}=-\omega_{0}^{1} \wedge \omega_{1}^{0}-\omega_{n+2}^{2} \wedge \omega_{1}^{2}-\omega_{n+2}^{0} \wedge \omega_{0}^{n+2}, \\
& d \omega_{j}^{2}=-\omega_{0}^{2} \wedge \omega_{n+1}^{3}-\omega_{1}^{2} \wedge \omega_{n+2}^{3}-\omega_{k}^{2} \wedge \omega_{j}^{k}-\omega_{n+1}^{2} \wedge \omega_{0}^{\jmath}-\omega_{n+2}^{2} \wedge \omega_{1}^{\jmath}, \\
& d \omega_{0}^{2}=-\omega_{0}^{i} \wedge \omega_{0}^{0}-\omega_{1}^{2} \wedge \omega_{0}^{1}-\omega_{j}^{2} \wedge \omega_{0}^{j}-\omega_{n+2}^{2} \wedge \omega_{0}^{n+2}, \\
& d \omega_{1}^{2}=-\omega_{0}^{2} \wedge \omega_{1}^{0}-\omega_{1}^{2} \wedge \omega_{1}^{1}-\omega_{j}^{2} \wedge \omega_{1}^{3}+\omega_{n+1}^{2} \wedge \omega_{0}^{n+2}, \\
& d \omega_{0}^{n+2}=\left(\omega_{0}^{0}+\omega_{1}^{1}\right) \wedge \omega_{0}^{n+2}-\omega_{1}^{2} \wedge \omega_{0}^{2} .
\end{aligned}
$$

Proof. Let $\bar{\omega}=s^{-1} d s, s=\left(s_{\beta}^{\alpha}\right)$. Then it is obvious that $\bar{\omega}$ is left invariant, $\bar{\omega}_{\beta}^{\alpha}=\omega_{\beta}^{\alpha}$ at $i d \in G$ and

$$
d \bar{\omega}_{\beta}^{\alpha}=-\bar{\omega}_{\gamma}^{\alpha} \wedge \bar{\omega}_{\beta}^{r} .
$$

Noting the relation among $\bar{\omega}_{\beta}^{\alpha}$ obtained in $\S 1$, we get the lemma.

q.e.d.

A Cartan connection $(P, \omega)$ of type $G / G^{\prime}$ over a manifold $M$ with $\operatorname{dim} M=$ $\operatorname{dim} G / G^{\prime}$, is by definition 
C1) $P$ is a principal fiber bundle over $M$ with structure group $G^{\prime}$,

C2) $\omega$ is a $g$-valued 1 -form on $P$ satisfying

(i) If $\omega(X)=0$, then $X=0$.

(ii) $R_{a}^{*} \omega=A d\left(a^{-1}\right) \omega, a \in G^{\prime}$.

(iii) $\omega\left(A^{*}\right)=A, A \in \mathrm{g}^{\prime}$.

In our case, let $(P, \omega)$ be a Cartan connection of type $G / G^{\prime}$ with $\theta=\omega_{-2}+\omega_{-1}$, where $\omega_{p}$ denotes the $g_{p}$-component of $\omega$. We put $\theta^{r}, \omega_{\nu}^{\mu}$, and $\omega_{\gamma}$, the components of $\omega$ with respect to the base of $g$ given above. Then the structure equations of $\omega$ are given by

$$
\begin{aligned}
& d \theta^{1}=-\left(\omega_{0}^{0}+\omega_{1}^{1}\right) \wedge \theta^{1}-\sum_{\imath} \theta^{2} \wedge \theta^{i}+\Omega^{1}, \\
& d \theta^{2}=\omega_{i} \wedge \theta^{1}-\omega_{j}^{2} \wedge \theta^{\jmath}+\theta^{2} \wedge \omega_{0}^{0}+\theta^{i} \wedge \omega_{1}^{0}+\Omega^{2}, \\
& d \theta^{\bar{i}}=-\omega_{i} \wedge \theta^{1}-\omega_{j}^{2} \wedge \theta^{\bar{j}}+\theta^{2} \wedge \omega_{0}^{1}+\theta^{\bar{i}} \wedge \omega_{1}^{1}+\Omega^{\bar{i}}, \\
& d \omega_{0}^{0}=-\omega_{1}^{0} \wedge \omega_{0}^{1}-\theta^{2} \wedge \omega_{i}-\theta^{1} \wedge \omega_{1}+\Omega_{0}^{0}, \\
& d \omega_{1}^{0}=-\left(\omega_{0}^{0}-\omega_{1}^{1}\right) \wedge \omega_{1}^{0}-\theta^{2} \wedge \omega_{i}+\Omega_{1}^{0}, \\
& d \omega_{0}^{1}=\left(\omega_{0}^{0}-\omega_{1}^{1}\right) \wedge \omega_{0}^{1}-\theta^{i} \wedge \omega_{i}+\Omega_{0}^{1}, \\
& d \omega_{1}^{1}=-\omega_{0}^{1} \wedge \omega_{1}^{0}-\theta^{\bar{i}} \wedge \omega_{i}-\theta^{1} \wedge \omega_{1}+\Omega_{1}^{1}, \\
& d \omega_{j}^{2}=-\omega_{i} \wedge \theta^{j}-\omega_{i} \wedge \theta^{j}-\omega_{k}^{2} \wedge \omega_{j}^{k}-\theta^{2} \wedge \omega_{j}-\theta^{i} \wedge \omega_{j}+\Omega_{j}^{2}, \\
& d \omega_{i}=-\omega_{i} \wedge \omega_{0}^{0}-\omega_{i} \wedge \omega_{0}^{1}+\omega_{i}^{\jmath} \wedge \omega_{j}-\theta^{i} \wedge \omega_{1}+\Omega_{\imath}, \\
& d \omega_{\bar{i}}=-\omega_{i} \wedge \omega_{1}^{0}-\omega_{\bar{i}} \wedge \omega_{1}^{1}+\omega_{i}^{\jmath} \wedge \omega_{j}+\theta^{2} \wedge \omega_{1}+\Omega_{\bar{i}}, \\
& d \omega_{1}=\left(\omega_{0}^{0}+\omega_{1}^{1}\right) \wedge \omega_{1}+\sum_{i} \omega_{i} \wedge \omega_{i}+\Omega_{1},
\end{aligned}
$$

where $i=2, \cdots, n, i \neq j$ and $\Omega^{r}, \Omega_{\nu}^{\mu}, \Omega_{\gamma}$ are the curvature forms of $\omega$.

In the same way as $[K N$, p. 220], we can write

$$
\Omega=\frac{1}{2} K \theta \wedge \theta \text {. }
$$

where $K(z) \in \mathfrak{g} \otimes \wedge^{2} \mathfrak{m}^{*}, z \in P$. For this curvature, Tanaka defines the *-curvature $K^{*}: P \rightarrow \mathfrak{g} \otimes \mathfrak{m}^{*}$ by

$$
K^{*}(z)(X)=\sum_{\gamma}\left[e_{\gamma}^{*}, K(z)\left(e_{\gamma}, X\right)\right], \quad X \in \mathfrak{m},
$$

up to scalar multiple $2(n+1)$ (see Lemma 3.2), where $\gamma$ is summed over $1, \cdots$, $n, \overline{2}, \cdots, \bar{n}$. In [T2] he calls a Cartan connection of type $G / G^{\prime}$ is normal when its *-curvatures vanish. On the other hand, he gives another definition of normal Cartan connections in [T3], and shows the meaning of the definition 
from the harmonic theory on the cohomology of Lie algebras. Here, we give and adopt the latter definition and apply it to the Lie contact structures.

For a simple graded Lie algebra $\mathrm{g}=\sum_{p \in \mathbf{Z}} \mathrm{g}_{p}$ of non-compact type with subalgebra $\mathfrak{m}=\sum_{p<0} \mathfrak{g}_{p}$, Tanaka constructed in [T3] a cochain complex $\left(C^{q}(\mathfrak{m}, \mathfrak{g}), \partial\right)$ where $C^{q}(\mathfrak{m}, \mathfrak{g})=g\left(\wedge^{q}\left(\mathfrak{m}^{*}\right)\right.$, and $\partial: C^{q} \rightarrow C^{q+1}$ is the coboundary operator (see also $[\mathrm{K}])$. Let $\partial^{*}: C^{q+1} \rightarrow C^{q}$ be the adjoint operator with respect to the metric $(X, Y)=-B(X, \sigma Y)$ defined by the Killing form $B$ and the involution $\sigma$ of $\mathrm{g}$. Explicitely, they are given by

$$
\begin{aligned}
(\partial c)\left(X_{1} \wedge \cdots\right. & \left.\wedge X_{q+1}\right)=\sum_{\imath}(-1)^{\imath+1}\left[X_{\imath}, c\left(X_{1} \wedge \cdots \wedge \hat{X}_{\imath} \wedge \cdots \wedge X_{q+1}\right)\right] \\
& +\sum_{\imath<j}(-1)^{\imath+\jmath} c\left(\left[X_{\imath}, X_{\jmath}\right] \wedge X_{1} \wedge \cdots \wedge \hat{X}_{\imath} \wedge \cdots \wedge \hat{X}_{\jmath} \wedge \cdots \wedge X_{q+1}\right), \\
(*) \quad\left(\partial^{*} c\right)\left(X_{1} \wedge \cdots \wedge X_{q-1}\right)=\sum_{\jmath}\left[e_{\jmath}^{*}, c\left(e_{\jmath} \wedge X_{1} \wedge \cdots \wedge X_{q-1}\right)\right] & \\
& +\frac{1}{2} \sum_{i, \jmath}(-1)^{\imath+1} c\left(\left[e_{J}^{*}, X_{\imath}\right]_{-} \wedge e_{\jmath} \wedge X_{1} \wedge \cdots \wedge \hat{X}_{\imath} \wedge \cdots \wedge X_{q-1}\right),
\end{aligned}
$$

where $c \in C^{q}(\mathfrak{m}, \mathfrak{g}), X_{1}, \cdots, X_{q+1} \in \mathfrak{m},\left[e_{\jmath}^{*}, X_{2}\right]_{\text {- denotes the }}$ m-component of $\left[e_{j}^{*}, X_{\imath}\right],\left\{e_{j}^{*}\right\}$ is the base of $\mathfrak{m}^{*}=\sum_{p>0} \mathfrak{g}_{p}$ dual to a base $\left\{e_{j}\right\}$ of $\mathfrak{m}$ with respect to $B$. Define

$$
\wedge_{i}^{q}=\Sigma g_{r_{1}}^{*} \wedge \cdots \wedge g_{r_{q}}^{*}
$$

where the summation is taken over $r_{1}, \cdots, r_{q}<0, \sum_{k=1}^{q} r_{k}=i$. Then we have $\wedge^{q}\left(\mathfrak{m}^{*}\right)=\sum_{i} \wedge_{i}^{q}$. Put

$$
C^{p, q}=\sum_{j} g_{j} \otimes \wedge_{j-p-q+1}^{q}
$$

Now, in our case, the curvature $\Omega=(1 / 2) K \theta \wedge \theta$ of a Cartan connection $\omega$ on $(P, \theta)$ is regarded as $K(z) \in C^{2}(\mathfrak{m}, \mathfrak{g}), z \in P$. Let $K^{p}$ be the $C^{p, 2}$-component of $K$. Then in [T3], $\omega$ is called normal if
1) $K^{-1}=0$,
2) $\partial^{*} K^{p}=0 \quad(p \geq 0)$.

We will express them using the components of $K$ with respect to the base defined above, i.e.

$$
K(z)\left(e_{\alpha}, e_{\beta}\right)=\sum_{\gamma} K_{\alpha \beta}^{\gamma}(z) e_{\gamma}+\sum_{\mu<\nu} K_{\nu \alpha \beta}^{\mu}(z) e_{\nu}^{\mu}+\sum_{\gamma} K_{\gamma \alpha \beta}(z) e_{\gamma}^{*},
$$

where $\gamma$ is summed over $1, \cdots, n, \overline{2}, \cdots, \bar{n},(\mu, \nu)=(0,0),(0,1),(1,0),(1,1),(i, j)$, $2 \leq i<j \leq n$. Now, note that

$$
\wedge^{2}(\mathfrak{m} *)=\wedge_{-4}^{2}+\wedge_{-3}^{2}+\wedge_{-2}^{2}
$$


since $\mathfrak{m}=\mathfrak{g}_{-2}+\mathfrak{g}_{-1}$. Noting also that $\operatorname{dim} \mathfrak{g}_{-2}=1$, we have

$$
\begin{aligned}
& K^{-1} \in C^{-1,2}=g_{-2} \otimes \wedge_{-2}^{2} \quad \longrightarrow \partial^{*} K^{-1} \in C^{0,1}=g_{-2} \otimes g_{-2}^{*}+g_{-1} \otimes g_{-1}^{*}, \\
& K^{0} \in C^{0,2}=\mathrm{g}_{-2} \otimes \wedge \stackrel{2}{2}_{3}+\mathrm{g}_{-1} \otimes \wedge \stackrel{2}{2}_{2} \longrightarrow \partial^{*} K^{0} \in C^{1,1}=\mathrm{g}_{-1} \otimes \mathrm{g}_{-2}^{*}+\mathrm{g}_{0} \otimes \mathrm{g}_{-1}^{*}, \\
& K^{1} \in C^{1,2}=\mathfrak{g}_{-1} \otimes \wedge_{-3}^{2}+g_{0} \otimes \wedge_{-2}^{2} \longrightarrow \partial^{*} K^{1} \in C^{2,1}=g_{0} \otimes g_{-2}^{*}+g_{1} \otimes g_{-1}^{*}, \\
& K^{2} \in C^{2,2}=g_{0} \otimes \wedge_{-3}^{2}+g_{1} \otimes \wedge_{-2}^{2} \quad \longrightarrow \partial^{*} K^{2} \in C^{3,1}=g_{1} \otimes g_{-2}^{*}+g_{2} \otimes g_{-1}^{*}, \\
& K^{3} \in C^{3,2}=\mathfrak{g}_{1} \otimes \wedge \perp_{-3}^{2}+\mathfrak{g}_{2} \otimes \wedge_{-2}^{2} \quad \longrightarrow \partial^{*} K^{3} \in C^{4,1}=\mathfrak{g}_{2} \otimes \mathfrak{g}_{-2}^{*}, \\
& K^{4} \in C^{4,2}=\mathrm{g}_{2} \otimes \wedge_{-3}^{2} \quad \longrightarrow \partial^{*} K^{4} \in C^{5,1}=0 .
\end{aligned}
$$

On the other hand, since we have

$$
\begin{array}{ll}
{\left[e_{1}^{*}, X\right]_{-}=0,} & X \in \mathfrak{m}, \\
{\left[e_{\jmath}^{*}, e_{1}\right]_{-}=e_{j},} & {\left[e_{\jmath}^{*}, \mathfrak{g}_{-1}\right]_{-}=0,} \\
{\left[e_{\jmath}^{*}, e_{1}\right]_{-}=-e_{\jmath},} & {\left[e_{\jmath}^{*}, \mathfrak{g}_{-1}\right]_{-}=0,}
\end{array}
$$

the second term of the right hand side of $\left(^{*}\right)$ where $c=K^{p}$ is written as

$$
-\sum_{j} K^{p}\left(e_{\jmath}, e_{j}\right) \text { for } X=e_{1} \text {, and } 0 \text { for } X \in g_{-1} \text {. }
$$

Therefore, using the $g_{p}$-component $K_{p}^{*}$ of the $*$-curvature, we get

and

$$
\left(\partial^{*} K^{p}\right)(X)=K_{p}^{*}(X), \quad X \in \mathrm{g}_{-1},
$$

$$
\left(\partial^{*} K^{p}\right)\left(e_{1}\right)=K_{p-1}^{*}\left(e_{1}\right)-\sum_{j=2}^{n} K_{p-1}\left(e_{j}, e_{j}\right)
$$

Now we have

Proposition 3.4. A Cartan connection $\omega$ on $(P, \theta)$ is normal in the sense [T3] if and only if

$$
\begin{aligned}
& K^{-1}=0 \leftrightarrow K_{i \jmath}^{1}=K_{i j}^{1}=K_{i j}^{1}=0, \\
& \left(\partial^{*} K^{0}\right)\left(e_{1}\right)=0 \leftrightarrow K_{1 j}^{1}-\sum_{\imath} K_{i i}^{j}=0, \quad K_{j 1}^{1}-\sum_{\imath} K_{i i}^{j}=0, \\
& \left(\partial^{*} K^{0}\right)\left(g_{-1}\right)=0
\end{aligned}
$$

$\leftrightarrow \sum_{\imath} K_{j i}^{i}=K_{1 \jmath}^{1}, \sum_{\imath} K_{j i}^{\bar{j}}=0, \sum_{\imath} K_{j \bar{j}}^{i}=0, \sum_{\imath} K_{j \bar{i}}^{\bar{i}}=K_{1 \jmath}^{1}, K_{j i}^{k}+K_{j i}^{\bar{k}}=K_{j k}^{i}+K_{j \bar{k}}^{\bar{i}_{\bar{k}}}(i \neq k)$, $\sum_{i} K_{j i}^{\imath}=K_{1 \bar{j}}^{1}, \sum_{i} K_{j i}^{i}=0, \sum_{i} K_{\bar{j} i}^{i}=0, \sum_{i} K_{j \bar{i}}^{\bar{i}}=K_{1 \bar{j}}^{1}, K_{j i}^{k}+K_{j \bar{i}}^{\bar{k}}=K_{\bar{j} k}^{\imath}+K_{\bar{j} \bar{i}}^{\bar{i}}(i \neq k)$, 


$$
\left(\partial^{*} K^{1}\right)\left(e_{1}\right)=0 \leftrightarrow \sum_{i}\left(K_{1 i}^{i}-K_{0 i \bar{i}}^{0}\right)=0, \sum_{i}\left(K_{1 i}^{i}-K_{1 i \bar{i}}^{0}\right)=0, \sum_{i}\left(K_{1 i}^{\bar{i}}-K_{0 i \bar{i}}^{1}\right)=0,
$$$$
\sum_{\imath}\left(K_{1 \bar{i}}^{\bar{i}}-K_{1 i \bar{i}}^{1}\right)=0, K_{1 k}^{j}-K_{1 j}^{k}+K_{1 \bar{k}}^{j}-K_{1 j}^{\bar{k}}-\sum_{\imath} K_{k \imath \bar{\imath}}^{\jmath}=0
$$

$$
\left(\partial * K^{1}\right)\left(g_{-1}\right)=0
$$

$$
\begin{aligned}
\leftrightarrow & -K_{1 j}^{i}+K_{0 \imath j}^{0}+K_{0 i j}^{1}-\sum_{k} K_{k k \jmath}^{i}=0, K_{1 j}^{i}+K_{1 \imath j}^{0}+K_{1 \bar{i} j}^{1}-\sum_{k} K_{k \bar{k} \jmath}^{i}=0, \\
& -K_{1 j}^{\bar{i}}+K_{0 \imath \bar{j}}^{0}+K_{0 i \bar{j}}^{1}-\sum_{k} K_{k k j}^{i}=0, K_{1 j}^{i}+K_{1 i j}^{0}+K_{1 \bar{j} j}^{1}-\sum_{k} K_{k \bar{k} j}^{i}=0,
\end{aligned}
$$

(3.7) $\quad\left(\partial^{*} K^{2}\right)\left(e_{1}\right)=0$

$$
\leftrightarrow K_{0 i 1}^{0}+K_{0 \bar{i}_{1}}^{1}-\sum_{k}\left(K_{k k 1}^{i}+K_{i k \bar{k}}\right)=0, K_{1 i 1}^{0}+K_{1 \bar{i} 1}^{1}-\sum_{k}\left(K_{k \bar{k} 1}^{i}+K_{\bar{i}_{k} \bar{k}}\right)=0,
$$

(3.8) $\quad\left(\partial^{*} K^{2}\right)\left(g_{-1}\right)=0$

$$
\leftrightarrow\left(K_{01 j}^{0}+K_{11 j}^{1}\right)-\sum_{i}\left(K_{\bar{i} i j}-K_{i \bar{\imath} \jmath}\right)=0,\left(K_{01 \bar{j}}^{0}+K_{11 \bar{j}}^{1}\right)-\sum_{\imath}\left(K_{\bar{i} i j}-K_{i \bar{i} j}\right)=0,
$$

(3.9) $\quad\left(\partial^{*} K^{3}\right)\left(e_{1}\right)=0 \leftrightarrow \sum_{\imath}\left(-K_{\bar{i} i_{1}}+K_{\imath \bar{i}_{1}}-K_{12 i}\right)=0$.

Proof. Since $K^{-1} \in C^{-1,2}=\mathfrak{g}_{-2} \otimes \wedge_{-2}^{2}$, we have $K^{-1}=0 \leftrightarrow K_{-2}(X, Y)=0, X, Y$ $\in \mathfrak{g}_{-1} \leftrightarrow(3.2)$. Now, we calculate $*$-curvature $K^{*}$ using the table of Lemma 3.1:

$$
\begin{aligned}
& K_{-1}^{*}\left(e_{1}\right)=\sum_{i}\left(\left[e_{i}^{*}, K_{-2}\left(e_{2}, e_{1}\right)\right]+\left[e_{2}^{*}, K_{-2}\left(e_{i}, e_{1}\right)\right]\right) \\
& =\sum_{i}\left\{\left[e_{\imath}^{*}, K_{i 1}^{1} e_{1}\right]+\left[e_{\imath}^{*}, K_{i 1}^{1} e_{1}\right]\right\} \\
& =\sum_{i}\left(K_{i 1}^{1} e_{i}-K_{\bar{i} 1}^{1} e_{i}\right), \\
& K_{-1}^{*}\left(e_{j}\right)=\sum_{i}\left(K_{i j}^{1} e_{i}-K_{i j}^{1} e_{i}\right), K_{-1}\left(e_{j}\right)=\sum_{i}\left(K_{i j}^{1} e_{i}-K_{i j}^{1} e_{\imath}\right) \\
& K_{0}^{*}\left(e_{1}\right)=\Sigma\left[e_{i}^{*}, K_{-1}\left(e_{2}, e_{1}\right)\right]+\Sigma\left[e_{i}^{*}, K_{-1}\left(e_{i}, e_{1}\right)\right] \\
& =\sum_{i, j}\left\{K_{1 i}^{j}\left(\delta_{j}^{i} e_{0}^{0}+e_{\imath}^{j}\right)+K_{1 i}^{j}\left(\delta_{j}^{i} e_{0}^{1}\right)+K_{1 i}^{j} \delta_{j}^{i} e_{1}^{0}+K_{1 \bar{i}}^{j}\left(\delta_{j}^{i} e_{1}^{1}+e_{\imath}^{j}\right)\right\} \\
& =\sum_{i}\left\{K_{1 i}^{i} e_{0}^{0}+K_{1 i}^{\bar{i}} e_{0}^{1}+K_{1 i}^{i} e_{1}^{0}+K_{1 i}^{\bar{i}} e_{1}^{1}\right\}+\sum_{i, j}\left(K_{1 i}^{\jmath}+K_{1 i}^{j}\right) e_{\imath}^{\jmath}, \\
& K_{0}^{*}\left(e_{j}\right)=\left[e_{1}^{*}, K_{-2}\left(e_{1}, e_{j}\right)\right]+\Sigma\left[e_{i}^{*}, K_{-1}\left(e_{i}, e_{j}\right)\right]+\left[e_{i}^{*}, K_{-1}\left(e_{i}, e_{j}\right)\right] \\
& =-K_{1 j}^{1} E+\sum_{\imath, k}\left\{K_{j i}^{k}\left(\delta_{k}^{i} e_{0}^{0}+e_{\imath}^{k}\right)+K_{j i}^{\bar{k}}\left(\delta_{k}^{i} e_{0}^{1}\right)+K_{j i}^{k}\left(\delta_{k}^{i} e_{0}^{0}\right)+K_{j i}^{\bar{k}} \bar{i}\left(\delta_{k}^{i} e_{1}^{1}+e_{\imath}^{k}\right)\right\} \\
& =\left(\sum_{\imath} K_{j i}^{i}-K_{1 j}^{1}\right) e_{0}^{0}+\sum\left\{K_{j i}^{\bar{j}} e_{0}^{1}+K_{j i}^{i} e_{1}^{0}\right\}+\left(\sum K_{j \bar{i}}^{\bar{i}}-K_{1 j}^{1}\right) e_{1}^{1}+\sum_{i, k}\left(K_{j i}^{k}+K_{j i}^{\bar{k}}\right) e_{\imath}^{k}, \\
& K_{0}^{*}\left(e_{j}\right)=-K_{1 j}^{1} E+\sum_{\imath, k}\left\{K_{j i}^{k}\left(\delta_{k}^{i} e_{0}^{0}+e_{\imath}^{k}\right)+K_{j i}^{\bar{k}}\left(\delta_{i}^{k} e_{0}^{1}\right)+K_{\bar{j} i}^{k}\left(\delta_{i}^{k} e_{1}^{0}\right)+K_{j i}^{\bar{k}}\left(\delta_{i}^{k} e_{1}^{1}+e_{\imath}^{k}\right)\right\} \\
& =\left(\sum_{\imath} K_{j i}^{2}-K_{1 j}^{1}\right) e_{0}^{0}+\sum\left\{K_{j i}^{i} e_{0}^{1}+K_{j i}^{i} e_{1}^{0}\right\}+\left(\sum K_{j i}^{i}-K_{1 j}^{1}\right) e_{1}^{1}+\sum_{i, k}\left(K_{j i}^{k}+K_{j i}^{\bar{k}}\right) e_{\imath}^{k},
\end{aligned}
$$




$$
\begin{aligned}
& K_{1}^{*}\left(e_{1}\right)=\sum_{\imath}\left\{\left[e_{\imath}^{*}, K_{0}\left(e_{\imath}, e_{1}\right)\right]+\left[e_{i}^{*}, K_{0}\left(e_{i}, e_{1}\right)\right]\right\} \\
& =\sum_{i}\left\{K_{0 i 1}^{0} e_{\imath}^{*}+K_{1 i 1}^{0} e_{i}^{*}+\frac{1}{2} \sum_{j, k} K_{j i 1}^{k}\left(\delta_{i}^{k} e_{j}^{*}-\delta_{i}^{\jmath} e_{k}^{*}\right)\right. \\
& \left.+K_{0 i \overline{1} 1}^{1} e_{\imath}^{*}+K_{1 \bar{i} 1}^{1} e_{i}^{*}+\frac{1}{2} \sum_{j, k} K_{j \bar{i} 1}^{k}\left(\delta_{i}^{k} e_{j}^{*}-\delta_{i}^{\jmath} e_{\bar{k}}^{*}\right)\right\} \\
& =\sum_{\imath}\left\{\left(K_{0 i 1}^{0}+K_{0 \bar{i}_{1}}^{1}-\sum_{k} K_{k k 1}^{i}\right) e_{\imath}^{*}+\left(K_{1 i 1}^{0}+K_{1 i_{1} 1}^{1}-\sum_{k} K_{k \bar{k} 1}^{i}\right) e_{i}^{*},\right. \\
& K_{1}^{*}\left(e_{\jmath}\right)=\left[e_{1}^{*}, K_{-1}\left(e_{1}, e_{\jmath}\right)\right]+\sum_{\imath}\left\{\left[e_{\imath}^{*}, K_{0}\left(e_{\imath}, e_{\jmath}\right)\right]+\left[e_{i}^{*}, K_{0}\left(e_{i}, e_{j}\right)\right]\right\} \\
& =\sum_{\imath}\left\{\left(-K_{1 j}^{\bar{i}}+K_{0 \imath j}^{0}+K_{0 i \bar{j}}^{1}-\sum_{k} K_{k k \jmath}^{i}\right) e_{\imath}^{*}+\left(K_{1 j}^{i}+K_{1 \imath j}^{0}+K_{1 \bar{\imath} j}^{1}-\sum_{k} K_{k \bar{k} \jmath}^{i}\right) e_{\imath}^{*},\right. \\
& K_{1}^{*}\left(e_{j}\right)=\sum_{\imath}\left(-K_{1 j}^{i}+K_{0 i j}^{0}+K_{0 i \bar{j}}^{1}-\sum_{k} K_{k k j}^{i}\right) e_{\imath}^{*}+\left(K_{1 j}^{i}+K_{1 \imath \bar{j}}^{0}+K_{1 i \bar{j}}^{1}-\sum_{k} K_{k \bar{k} \bar{j}}^{i}\right) e_{\imath}^{*}, \\
& K_{2}^{*}\left(e_{1}\right)=\sum_{\imath}\left\{\left[e_{\imath}^{*}, K_{1}\left(e_{\imath}, e_{1}\right)\right]+\left[e_{\imath}^{*}, K_{1}\left(e_{\bar{\imath}}, e_{1}\right)\right]\right\} \\
& =\sum_{i}\left(-K_{\bar{i} i 1}+K_{i \bar{i} 1}\right) e_{1}^{*}, \\
& K_{2}^{*}\left(e_{j}\right)=\left[e_{1}^{*}, K_{0}\left(e_{1}, e_{\jmath}\right)\right]+\sum_{\imath}\left\{\left[e_{\imath}^{*}, K_{1}\left(e_{\imath}, e_{\jmath}\right)\right]+\left[e_{\imath}^{*}, K_{1}\left(e_{i}, e_{\jmath}\right)\right]\right\} \\
& =\left(K_{01 j}^{0}+K_{11 j}^{1}\right) e_{1}^{*}-\sum_{i}\left(K_{\bar{i} i j}-K_{i \bar{\imath} \jmath}\right) e_{1}^{*}, \\
& K_{2}^{*}\left(e_{\bar{j}}\right)=\left\{K_{01 \bar{j}}^{0}+K_{11 \bar{j}}^{1}-\sum_{\imath}\left(K_{i i j}-K_{i \bar{i} j}\right)\right\} e_{1}^{*},
\end{aligned}
$$

Now, for $\partial^{*} K^{0}=0$, noting that

$$
\sum_{i} K_{-1}\left(e_{\imath}, e_{i}\right)=\sum_{i, j}\left(K_{i \bar{i}}^{j} e_{j}+K_{i \bar{i}}^{\bar{j}} e_{j}\right),
$$

we get (3.3). (3.4) is clear. For $\partial^{*} K^{1}=0$, from

$$
\sum_{i} K_{0}\left(e_{i}, e_{i}\right)=\sum_{i}\left(K_{0 i i}^{0} e_{0}^{0}+K_{1 i i}^{0} e_{1}^{0}+K_{0 i i}^{1} e_{0}^{1}+K_{1 \imath i}^{1} e_{1}^{1}+\frac{1}{2} \sum_{j, k} K_{j i i}^{k} e_{j}^{k}\right),
$$

we obtain (3.5) and (3.6). Similarly we get (3.7), (3.8) and (3.9). q.e.d.

LEMMMA 3.5. When $K_{-2}=0$, (3.2) (3.4) are equivalent with

$$
\begin{aligned}
& \sum_{\imath} K_{j i}^{i}=0, \sum_{\imath} K_{j \imath}^{\tilde{i}}=0, \sum_{\imath} K_{j i}^{i}=0, \sum_{\imath} K_{j i}^{i}=0, K_{j i}^{k}+K_{j i}^{\bar{k}}=K_{j k}^{i}+K_{j \bar{k}}^{\bar{i}}(\imath \neq k), \\
& \sum_{i} K_{j i}^{i}=0, \sum_{\imath} K_{j i}^{\bar{i}}=0, \sum_{\imath} K_{j i}^{i}=0, \sum_{\imath} K_{j i}^{\bar{i}}=0, K_{j i}^{k}+K_{j \bar{j}}^{\bar{k}}=K_{j k}^{\imath}+K_{j \bar{k}}^{\bar{i}}(i \neq k) .
\end{aligned}
$$

Proof. For convenience, denote by $(3 . j)_{k}$ the $k$-th formula in $(3 . j)$. Summing up $i=j$ in $(3.4)_{10}$ and $(3.4)_{5}$, we get 


$$
\sum_{i} K_{\bar{i} \imath}^{k}=\sum_{\imath} K_{i_{k}}^{\imath}+\sum_{\imath} K_{\bar{i} \bar{k}}^{\bar{i}}, \quad \sum_{i} K_{\imath \bar{i}}^{\bar{k}}=\sum_{\imath} K_{i k}^{i}+\sum_{\imath} K_{i \bar{k}}^{\bar{i}}
$$

Noting $(3.4)_{3}$ and $(3.4)_{9},(3.4)_{1}$ and $(3.4)_{7}$, we get the lemma.

q.e.d.

\section{§4. Tanaka theory.}

When $(P, \omega)$ is a Cartan connection of type $G / G^{\prime}$ over a manifold $M$, a principal fibre bundle $(\tilde{P}, \tilde{\theta})$ over $M$ with structure group $\tilde{G}$ is defined by $\widetilde{P}=$ $P / \operatorname{ker} \rho, \tilde{\theta}$ is a unique $\mathfrak{m}$-valued 1 -form on $\tilde{P}$ with $\theta=\tilde{\rho}^{*} \tilde{\theta}$, where $\theta=\omega_{-2}+\omega_{-1}$ and $\tilde{\rho}: P \rightarrow \widetilde{P}$ is the bundle homomorphism corresponding to the homomorphism $\rho: G^{\prime} \rightarrow \tilde{G}$. Then $(\tilde{P}, \tilde{\theta})$ is a $\tilde{G}$-structure on $M$ and every isomorphism $\varphi:(P, \omega)$ $\rightarrow\left(P^{\prime}, \omega^{\prime}\right)$ induces an isomorphism $\tilde{\varphi}:(\tilde{P}, \tilde{\theta}) \rightarrow\left(\tilde{P}^{\prime}, \tilde{\theta}^{\prime}\right)[\mathrm{T} 2,1.2]$. When $K^{-1}=0$, the $\tilde{G}$-structure is of type $\mathfrak{m}$, which is the case when $(P, \omega)$ is a normal Cartan connection.

The purpose of this section is to construct a normal Cartan connection $(P, \omega)$ of type $G / G^{\prime}$ corresponding to a given $\tilde{G}$-structure $(\widetilde{P}, \tilde{\theta})$ of type $\mathfrak{m}$, uniquely up to isomorphism.

To begin with, let $\mathfrak{h}_{0}=\left\{X \in \mathfrak{g}_{0} \mid \rho_{-2}(X)=0\right\}=\left\{\left(\begin{array}{llr}a & 0 & 0 \\ 0 & e & 0 \\ 0 & 0 & -{ }^{t} a\end{array}\right) \in \mathfrak{g}_{0}, \operatorname{Tr} a=0\right\}$, and let $H_{0}$ be a closed subgroup of $G_{0}$ corresponding to $\mathfrak{h}_{0}$, i. e. $H_{0}=\left\{a \in G_{0} \mid \operatorname{det} \rho(a)= \pm 1\right\}$ $=\left\{\left(\begin{array}{ccc}A & 0 & 0 \\ 0 & g & 0 \\ 0 & 0 & { }^{t} A^{-1}\end{array}\right)\right.$, det $\left.A= \pm 1\right\}$. Note that $\mathfrak{g}_{0}=\boldsymbol{R} E+\mathfrak{h}_{0}$, where $E=\left(\begin{array}{ccc}-I_{2} & 0 & 0 \\ 0 & 0 & 0 \\ 0 & 0 & I_{2}\end{array}\right)$. Moreover let $\mathfrak{h}=\mathfrak{g}_{-2}+\mathfrak{g}_{-1}+\mathfrak{h}_{0}$, with a corresponding group $H=G_{-} \cdot H_{0}$, where $G_{-}$ is the Lie subgroup of $G$ generated by the subalgebra $\mathfrak{m}$. Then since $\tilde{G} / H_{0}$ is homeomorphic to $\boldsymbol{R} \times \mathrm{g}_{1}$, there exists an $H_{0}$-reduction $(Q, \zeta)$ of $(\tilde{P}, \tilde{\theta})$. In the riemannian case, this is given by, for instance, $Q=P_{g} \times_{o(n-1)} H_{0}$.

Now, we introduce the theory in [T2] on the uniqueness of the normal Cartan connection on a given $\tilde{G}$-structure $(\tilde{P}, \tilde{\theta})$ step by step.

Step 1 [T2, Proposition 6.1]. Take an $H_{0}$-reduction $(Q, \zeta)$ satisfying

$$
d \zeta_{-2}+\frac{1}{2}\left[\zeta_{-1}, \zeta_{-1}\right]=0 .
$$

Step 2. Extend $(Q, \zeta)$ naturally to a $G^{\prime}$-bundle $(P, \theta)$ by

$$
P=Q \times{ }_{H_{0}} G^{\prime} .
$$

Step 3. Let $\chi$ be any Cartan connection of type $H / H_{0}$ on $(Q, \zeta)$, compatible with $\zeta$.

Step 4 [T2, Lemma 1.6]. Extend $\chi$ uniquely to a Cartan connection $\omega$ of type $G / G^{\prime}$ on $(P, \theta)$.

Stcp 5. Noting that $K_{-2}=0$ for $\omega$ in Step 4 , we can make an $E$-system 
$\left(P, \theta, \omega_{E}\right)$ from $\omega$.

Step 6 [T2, Proposition $7.1(1)]$. This $E$-system $\left(P, \theta, \omega_{E}\right)$ induces the original $\tilde{G}$-structure $(\tilde{P}, \tilde{\theta})$ of type $\mathfrak{m}$.

Step 7 [T2, Proposition 7.1 (2)]. If two $\tilde{G}$-structures of type $\mathfrak{m}$ induced by two $E$-systems are isomorphic, then the two $E$-systems are isomorphic. In this sense, the $E$-system in Step 6 is unique.

Step 8 [T2, Proposition 7.2]. For a given $E$-system $\left(P, \theta, \omega_{E}\right)$, there exists a unique normal Cartan connection of type $G / G^{\prime},(P, \omega)$.

Step 9 [T2, 7.2]. Following Step 1-8, we can construct a normal Cartan connection $(P, \omega)$ corresponding uniquely to a given $\tilde{G}$-structure of type $G / G^{\prime}$ from a specified $\chi$ on $(Q, \zeta)$ of Step 1 .

Now we briefly investigate each step: we prove Step 1 in $\S 5$. Step 2-4 are elementary. An $E$-system $(P, \theta, \alpha)$ is by definition,

E0) $P$ is a principal fibre bundle over a manifold of dimension $2 n-1$ with structure group $G^{\prime}, \theta$ is an $\mathfrak{m}$-valued 1 -form on $P$ and $\alpha$ is an $\boldsymbol{R} E$-valued 1 form on $P$.

E1) $\theta(X)=0$ if and only if $X$ is vertical, $X \in T P$.

E2) $R_{a}^{*} \theta=\rho\left(a^{-1}\right) \theta$ for $a \in G^{\prime}$.

E3) $R_{a}^{*} \alpha=\alpha+\left(A d\left(a^{-1}\right) \theta\right)_{E}$ for $a \in G^{\prime}$, where ( $)_{E}$ denotes the $E$-component.

E4) $\alpha\left(X^{*}\right)=X_{E}$ for $X \in \mathfrak{g}^{\prime}$.

E5) $d \theta_{-2}+\frac{1}{2}\left[\theta_{-1}, \theta_{-1}\right]+\left[\alpha, \theta_{-2}\right]=0$.

Note that $\theta_{-2}=\theta^{1} e_{1}, \theta_{-1}=\theta^{2} e_{i}+\theta^{\bar{i}} e_{\bar{i}}$. Now, Step 5 is obvious since $K_{-2}=0$ is a consequence of (4.1). As for Step 6, since we have $\rho(x)=x$ for $x \in Q$, it follows $\iota^{*} \theta=\zeta=\iota^{*}\left(\tilde{\rho}^{*} \tilde{\theta}\right)$ where $\iota: Q \rightarrow P$ is the inclusion map, we get $\theta=\tilde{\rho}^{*} \tilde{\theta}$. Step 7 is important and we show it in detail. By a simple calculation, we obtain:

LEMMA 4.1. Let $x \in g_{2}$ and let $e_{1}, \cdots, e_{n}, e_{\overline{2}}, \cdots, e_{\bar{n}}$ be the base of $\mathfrak{m}$ given in $\S 1$ and 3 . Then

$$
\begin{aligned}
& \left(\operatorname{Ad}(\exp x) e_{1}\right)_{E}=-\left[e_{1}, x\right], \\
& \left(\operatorname{Ad}(\exp x) e_{\gamma}\right)_{E}=0, \quad \gamma=i, i, 2 \leqq i \leqq n .
\end{aligned}
$$

Now, Let $(P, \theta, \alpha)$ (resp. $\left.\left(P^{\prime}, \theta^{\prime}, \alpha^{\prime}\right)\right)$ be an $E$-system on $M$ (resp. $M^{\prime}$ ) and let $(\tilde{P}, \tilde{\theta})$ (resp. $\left(\tilde{P}^{\prime}, \hat{\theta}^{\prime}\right)$ ) be the corresponding $\tilde{G}$-structure of type $\mathfrak{m}$ on $M$ (resp. $\left.M^{\prime}\right)$. We show that if $\tilde{\varphi}$ is an isomorphism $(\tilde{P}, \tilde{\theta}) \rightarrow\left(\tilde{P}^{\prime}, \tilde{\theta}^{\prime}\right)$, there is a unique isomorphism $\varphi:(P, \theta, \alpha) \rightarrow\left(P^{\prime}, \theta^{\prime}, \alpha^{\prime}\right)$ which induces the given $\tilde{\varphi}$. In fact, for the uniqueness; Let $\varphi_{1}$ and $\varphi_{2}:(P, \theta, \alpha) \rightarrow\left(P^{\prime}, \theta^{\prime}, \alpha^{\prime}\right)$ be two isomorphisms which induce the given isomorphism $\tilde{\varphi}$. We can find a unique map $\sigma: P \rightarrow G^{\prime}$ such that $\varphi_{2}(z)=\varphi_{1}(z) \sigma(z)$ for $z \in P$. Clearly $\sigma(z) \in \operatorname{Ker} \rho$, and so we may ex- 
press $\sigma(z)=\exp \tau(z), \tau: P \rightarrow g_{2}$. For $z \in P$ and $X \in T P$, we can decompose

$$
\varphi_{2 *} X=\left(R_{\sigma(z) *}\right) \varphi_{1 *} X+Y_{\varphi_{2}(z)}^{*},
$$

where $Y \in g_{2}$. Using Lemma 4.1 and E3), we can show

$$
\varphi_{2}^{*} \alpha^{\prime}=\varphi_{1}^{*} \alpha^{\prime}+\left[\varphi_{1}^{*} \theta_{-2}^{\prime}, \tau\right] .
$$

Since $\varphi_{i}^{*} \theta^{\prime}=\theta$ and $\varphi_{i}^{*} \alpha^{\prime}=\alpha, i=1,2$, we get $\left[\theta_{-2}, \tau\right]=0$ or $\tau=0$. Now, we construct the isomorphism $\varphi$ from any bundle isomorphism $\varphi_{1}: P \rightarrow P^{\prime}$ By the uniqueness, we may assume $P$ and $P^{\prime}$ are trivial. In order to be $\varphi^{*} \alpha^{\prime}=\alpha$, we must find a function $\tau: P \rightarrow g_{2}$ satisfying $\alpha-\varphi_{1}^{*} \alpha^{\prime}=\left[\theta_{-2}, \tau\right]$ (note (4.2)). Since we have $\left(\alpha-\varphi_{1}^{*} \alpha^{\prime}\right) \wedge \theta^{1}=0$ from E5) and $\varphi_{1}^{*} \theta^{\prime}=\theta$, we get $\alpha-\varphi_{1}^{*} \alpha^{\prime}=A \theta^{1} E$ for some function $A$ on $P$. Now, since $\left(\alpha-\varphi_{1}^{*} \alpha^{\prime}\right)(X)=A(z) \theta^{1}(X) E=\left[\theta^{1}(X) e_{1}, A(z) e_{1}^{*}\right]$ for $z \in P$, and $X \in T P$, we obtain $\tau=A e_{1}^{*}$. Because of $R_{a}^{*}\left(\alpha-\varphi_{1}^{*} \alpha^{\prime}\right)=\alpha-\varphi_{1}^{*} \alpha^{\prime}$, using $A d(a) e_{1}^{*} \in \mathrm{g}_{2}$ and $\left(A d\left(a^{-1}\right) E\right)_{E}=E$, we see that $A d(a) \tau(z a)=\tau(z)$ for $z \in P$ and $a \in G^{\prime}$. Then for $\sigma(z)=\exp \tau(z)$, we get $\sigma(z a)=a^{-1} \sigma(z) a$. Thus we obtain the desired bundle isomorphism $\varphi: P \rightarrow P^{\prime}$ by $\varphi(z)=\varphi_{1}(z) \sigma(z)$.

In the next section, we prove Step 1 and construct a normal Cartan connection from this $H_{0}$-reduction, proving Step 8 at the same time.

\section{$\S 5$. The construction.}

In this section, we prove the following three propositions to obtain the normal Cartan connection corresponding to a Lie contact structure $(\hat{P}, \tilde{\theta})$.

Proposition 5.1. For any $H_{0}$-reduction $\left(Q^{\prime}, \zeta^{\prime}\right)$ of $(\tilde{P}, \tilde{\theta})$, there exists an $H_{0^{-}}$ reduction $(Q, \zeta)$ with basic form $\zeta$, satisfying

$$
d \zeta_{-2}+\frac{1}{2}\left[\zeta_{-1}, \zeta_{-1}\right]=0
$$

Proposition 5.2. Let $n \geq 3$. Then on $(Q, \zeta)$ of Proposition 5.1 , there exists a unique Cartan connection $\chi$ of type $H / H_{0}$ satisfying $\zeta=\chi_{-2}+\chi_{-1}, T^{-1}=\partial^{*} T^{0}=$ $\left(\partial * T^{1}\right)\left(e_{1}\right)=0$, where $T$ denotes the curvature of $\chi$.

Proposition 5.3. Let $n \geq 3$. Extend $(Q, \zeta)$ of Proposition 5.1 to $a G^{\prime}$ principal bundle $P$ by

$$
P=Q \times{ }_{H} G^{\prime} .
$$

Then there exists a unique normal Cartan connection $(P, \omega)$ of type $G / G^{\prime}$ in the sense [T3], which induces the Lie contact structure $(\tilde{P}, \tilde{\theta})$.

Proof of Proposition 5.1. Let $(Q, \zeta)$ and $\left(Q^{\prime}, \zeta^{\prime}\right)$ be any two $H_{0}$-reductions of $(\tilde{P}, \tilde{\theta})$. Then, there exists a map $\sigma: Q \rightarrow \exp \boldsymbol{R} E \exp g_{1}$ such that $x \sigma(x) \in Q^{\prime}$, 
$x \in Q$. Note that an element $s=\left(s_{\beta}^{\alpha}\right)$ in $\exp \boldsymbol{R} E \exp g_{1}$ is written as

$$
\begin{aligned}
s & =\left(\begin{array}{lcc}
\lambda I_{2} & 0 & 0 \\
0 & I_{n-1} & 0 \\
0 & 0 & \frac{1}{\lambda} I_{2}
\end{array}\right)\left(\begin{array}{lll}
I_{2} & 0 & 0 \\
d & I_{n-1} & 0 \\
\frac{t}{2} d d & { }^{t} d & I_{2}
\end{array}\right) \\
& =\left(\begin{array}{lll}
\lambda I_{2} & 0 & 0 \\
d & I_{n-1} & 0 \\
\frac{t}{2 d} & \frac{{ }^{t} d}{2 \lambda} & \frac{1}{\lambda} I_{2}
\end{array}\right)
\end{aligned}
$$

and so from Lemma 1.3, we get

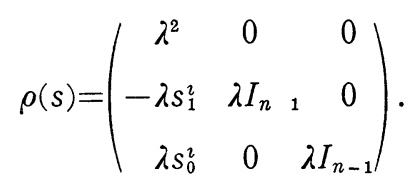

Now, since we have $\zeta^{\prime}=\sigma^{-1} \zeta, \zeta^{\prime}=\zeta^{\prime 1} e_{1}+\zeta^{\prime 2} e_{i}+\zeta^{\prime i} e_{i}$ and $\zeta=\zeta^{1} e_{1}+\zeta^{i} e_{i}+\zeta^{\bar{i}} e_{\bar{i}}$, putting $\sigma^{-1}=\rho(s)$, we obtain

$$
\begin{aligned}
\zeta^{\prime} & =\sigma^{-1} \zeta=\sigma^{-1}\left(\zeta^{1} e_{1}+\zeta^{i} e_{i}+\zeta^{\bar{i}} e_{i}\right) \\
& =\zeta^{1}\left(\lambda^{2} e_{1}-\lambda s_{1}^{2} e_{i}+\lambda s_{0}^{2} e_{i}\right)+\zeta^{i} \lambda e_{i}+\zeta^{i} \lambda e_{i} \\
& =\lambda^{2} \zeta^{1} e_{1}+\lambda\left(\zeta^{i}-s_{1}^{i} \zeta^{1}\right) e_{i}+\lambda\left(\zeta^{\bar{i}}+s_{0}^{i} \zeta^{1}\right) e_{i}
\end{aligned}
$$

and so

$$
\zeta^{\prime 1}=\lambda^{2} \zeta^{1}, \quad \zeta^{\prime 2}=\lambda\left(\zeta^{i}-s_{1}^{i \zeta^{1}}\right), \quad \zeta^{\prime i}=\lambda\left(\zeta^{\bar{i}}+s_{0}^{i \zeta^{1}}\right) .
$$

From the structure equation, we obtain

$$
\begin{aligned}
& d \zeta_{-2}=-\zeta^{i} \wedge \zeta^{\bar{i}}+\Omega^{1}, \\
& d \zeta_{-2}^{\prime}=-\zeta^{\prime 2} \wedge \zeta^{\prime i}+\Omega^{\prime 1},
\end{aligned}
$$

therefore

$$
\begin{aligned}
\Omega^{\prime 1}-\Omega^{1} & =d \zeta^{\prime 1}+\zeta^{\prime 2} \wedge \zeta^{\prime i}-d \zeta^{1}-\zeta^{i} \wedge \zeta^{i} \\
& =\left(d \lambda^{2}\right) \zeta^{1}+\lambda^{2}\left\{d \zeta^{1}+\left(\zeta^{i}-s_{1}^{i} \zeta^{1}\right) \wedge\left(\zeta^{\bar{i}}+s_{0}^{i} \zeta^{1}\right)\right\}-d \zeta^{1}-\zeta^{i} \wedge \zeta^{\bar{i}} \\
& =\left(d \lambda^{2}\right) \zeta^{1}+\left(\lambda^{2}-1\right)\left(d \zeta^{1}+\zeta^{i} \wedge \zeta^{\bar{i}}\right)+\lambda^{2}\left(-s_{0}^{i} \zeta^{1} \wedge \zeta^{i}-s_{1}^{i} \zeta^{1} \wedge \zeta^{i}\right)
\end{aligned}
$$

Now, since $(\tilde{P}, \tilde{\theta})$ is of type $\mathfrak{m}$, we can write

$$
\Omega^{\prime 1}=T^{1}{ }_{1 \gamma} \zeta^{1} \wedge \zeta^{\gamma}, \quad \gamma=2, \cdots, n, \overline{2}, \cdots, \bar{n} .
$$

Noting this fact, from an arbitrary $H_{0}$-reduction $\left(Q^{\prime}, \zeta^{\prime}\right)$ which exists by the reason stated in the beginning of $\S 4$, we construct the desired $(Q, \zeta)$ as follows: 
Define $a$ map $\tau: Q \rightarrow g_{1}$ by

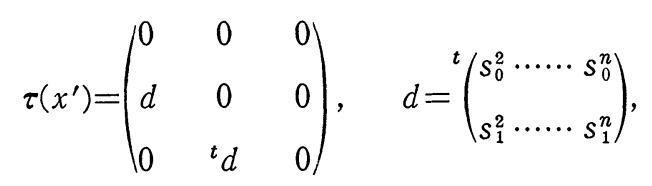

where $s_{0}^{2}$ and $s_{1}^{2}$ are given by

$$
s_{0}^{2}=-T^{\prime 1}{ }_{1 \imath}, \quad s_{1}^{2}=-T^{\prime 1}{ }_{1 i} .
$$

From $R_{a}^{*} T^{\prime}=A d\left(a^{-1}\right) T^{\prime}$ for $a \in H_{0}$, we get $\tau\left(x^{\prime} a\right)=A d\left(a^{-1}\right) \tau\left(x^{\prime}\right)$. Now, for $\sigma\left(x^{\prime}\right)$ $=\exp \tau\left(x^{\prime}\right)$, since $\sigma\left(x^{\prime} a\right)=a^{-1} \sigma\left(x^{\prime}\right) a$ and so $x^{\prime} a \sigma\left(x^{\prime} a\right)=x^{\prime} \sigma\left(x^{\prime}\right) a$ hold, we obtain a subbundle $(Q, \zeta)=\left\{x^{\prime} \sigma\left(x^{\prime}\right), x^{\prime} \in Q^{\prime}\right\}$. Now, from (5.2), where $\lambda=1$ for this $\sigma$, we get $(5.1)$ on $(Q, \zeta)$.

q.e.d.

Remark. In the Riemannian case, the basic form of $P_{g} \times_{o(n-1)} H_{0}$ satisfies (5.1) [M2].

Proof of Proposition 5.2. By (5.1) and $\chi_{0}^{0}+\chi_{1}^{1}=0$, note that a Cartan connection $(Q, \chi)$ of type $H / H_{0}$ such that $\zeta=\chi_{-2}+\chi_{-1}$ satisfies $T_{-2}=0$. Then by Lemma 3.2, we may check (3.4) and (3.5) for $\chi$ to satisfy $T^{-1}=\partial^{*} T^{0}=\left(\partial^{*} T^{1}\right)\left(e_{1}\right)$ $=0$.

Uniqueness: Let $\chi$ and $\chi^{\prime}$ be two Cartan connections satisfying the conditions in the proposition. Then $\chi_{-2}=\chi_{-2}^{\prime}, \chi_{-1}=\chi_{-1}^{\prime}$. Since $\chi_{0}-\chi_{0}^{\prime}=0$ on the fiber, we can write

$$
\chi_{\beta}^{\alpha}-\chi_{\beta}^{\prime \alpha}=A_{\beta \gamma}^{\alpha} \gamma^{\gamma},
$$

where $(\alpha, \beta)=(0,0),(0,1),(1,0),(1,1),(i, j), 2 \leq i<j \leq n, \chi_{0}^{0}+\chi_{1}^{1}=\chi_{0}^{\prime 0}+\chi_{1}^{\prime 1}=0$ and $\gamma$ is summed over $1, \cdots, n, \overline{2}, \cdots, \bar{n}$. Let $\Psi$ and $\Psi^{\prime}$ be the corresponding curvature forms. Then by the structure equations (3.1), we get

$$
\begin{aligned}
& \Psi^{\prime \imath}-\Psi^{i}=-A_{\jmath \gamma}^{2} \zeta^{\gamma} \wedge \zeta^{j}+\zeta^{i} \wedge A_{0 \gamma}^{0} \zeta^{\gamma}+\zeta^{\bar{i}} \wedge A_{1 \gamma}^{0} \zeta^{\gamma}, \\
& \Psi^{\prime i}-\Psi^{i}=-A_{\partial \gamma}^{2} \zeta^{\gamma} \wedge \zeta^{\bar{j}}+\zeta^{i} \wedge A_{0 \gamma}^{1} \zeta^{\gamma}+\zeta^{\bar{i}} \wedge A_{1 \gamma}^{1} \zeta^{\gamma} .
\end{aligned}
$$

Here and hereafter, $i, j, k$ are summed over $2, \cdots, n$, and $\gamma$ is summed over $1, \cdots, n, \overline{2}, \cdots, \bar{n}$, by using Einstein convention. Now, using the components $T_{\beta \gamma}^{\alpha}$ and $T_{\beta \gamma}^{\alpha}$ of $\Psi$ and $\Psi^{\prime}$, we can write the left hand side of (5.3) and (5.4),

$$
\frac{1}{2}\left(T_{\beta \gamma}^{\prime 2}-T_{\beta \gamma}^{i}\right) \zeta^{\beta} \wedge \zeta^{\gamma} \text { and } \frac{1}{2}\left(T_{\beta \gamma}^{\bar{i}}-T_{\beta \gamma}^{\bar{i}}\right) \zeta^{\beta} \wedge \zeta^{\gamma},
$$

where we may assume that $T_{\beta \gamma}^{2}=-T_{\gamma \beta}^{2}, T_{\beta \gamma}^{\prime}=-T_{\gamma \beta}^{\prime}$. For simplicity, put $\tilde{T}_{\beta \gamma}^{\alpha}=T_{\beta \gamma}^{\alpha}-T_{\beta \gamma}^{\alpha}$. Then we have

$$
\begin{aligned}
& \tilde{T}_{1 \jmath}^{i}=-A_{j 1}^{2}-A_{01}^{0} \delta_{\jmath}^{i}, \\
& \tilde{T}_{1 j}^{i}=-A_{11}^{0} \delta_{\jmath}^{2},
\end{aligned}
$$




$$
\begin{aligned}
& \widetilde{T}_{1 j}^{\bar{i}}=-A_{01}^{1} \delta_{j}^{i}, \\
& \widetilde{T}_{1 j}^{i}=-A_{j 1}^{2}-A_{11}^{1} \delta_{j}^{2}, \\
& \tilde{T}_{j k}^{i}=A_{j k}^{2}-A_{k j}^{2}+A_{0 k}^{0} \delta_{j}^{2}-A_{0 j}^{0} \delta_{k}^{2}, \\
& \tilde{T}_{j \bar{k}}^{i}=A_{j \bar{k}}^{2}+A_{0 \bar{k}}^{0} \delta_{j}^{i}-A_{1 j}^{0} \delta_{k}^{2}, \\
& \widetilde{T}_{\bar{j}_{\bar{k}}}^{2}=\delta_{j}^{2} A_{1 \bar{k}}^{0}-A_{1 j}^{0} \delta_{k}^{2}, \\
& \widetilde{T}_{j k}^{\bar{i}}=A_{0 k}^{1} \delta_{j}^{2}-A_{0 j}^{1} \delta_{k}^{2}, \\
& \tilde{T}_{j \bar{k}}^{\bar{i}}=-A_{k j}^{2}+A_{0 \bar{k}}^{1} \delta_{j}^{2}-A_{1 j}^{1} \delta_{k}^{2}, \\
& \widetilde{T}_{\bar{j} \bar{k}}^{\bar{i}}=A_{j \bar{k}}^{2}-A_{k \bar{j}}^{2}+A_{1 \bar{k}}^{1} \delta_{j}^{2}-A_{1 j}^{1} \delta_{k}^{2} .
\end{aligned}
$$

Note that $A_{0 \gamma}^{0}+A_{1 \gamma}^{1}=0, A_{\jmath \gamma}^{\imath}=-A_{i \gamma}^{\jmath}, A_{i \gamma}^{2}=0$. Then (3.4) implies

$$
\begin{aligned}
& 0=\Sigma \tilde{T}_{j i}^{i}=\Sigma A_{j i}^{i}+A_{0 j}^{0}-(n-1) A_{0 j}^{0} \longleftrightarrow \Sigma A_{j i}^{2}-(n-2) A_{0 j}^{0}=0 \\
& 0=\Sigma \widetilde{T}_{j i}^{\bar{i}}=A_{0 j}^{1}-(n-1) A_{0 j}^{1} \longleftrightarrow A_{0 j}^{1}=0 \\
& 0=\sum \widetilde{T}_{j \bar{i}}^{i}=\sum A_{j \bar{i}}^{i}+A_{0 j}^{0}-(n-1) A_{1 \jmath}^{0} \longleftrightarrow \sum A_{j \bar{i}}^{i}+A_{0 j}^{0}-(n-1) A_{1 \jmath}^{0}=0 \\
& 0=\Sigma \tilde{T}_{j i}^{i_{i}}=A_{0 j}^{1}-(n-1) A_{1 \jmath}^{1} \longleftrightarrow A_{0 j}^{1}-(n-1) A_{1 j}^{1}=0 \\
& 0=\tilde{T}_{j i}^{k}-\tilde{T}_{j k}^{i}+\left(\tilde{T}_{j \bar{i}}^{\bar{k}_{\bar{i}}-} \tilde{T}_{j \bar{k}}^{\bar{i}}\right)=\left\{\begin{array}{cl}
-4 A_{i j}^{k}-A_{k i}^{j}-A_{j k}^{i} & (i \neq j \neq k \neq i) \\
3 A_{k i}^{2}-A_{0 k}^{0}-A_{0 \bar{k}}^{1} & (i=j \neq k)
\end{array}\right. \\
& 0=\sum \tilde{T}_{i j}^{i}=(n-1) A_{0 j}^{0}-A_{1 \jmath}^{0} \longleftrightarrow(n-1) A_{0 j}^{0}-A_{1 \jmath}^{0}=0 \\
& 0=\Sigma \tilde{T}_{i j}^{\bar{i}}=-\sum A_{j i}^{2}+(n-1) A_{0 j}^{1}-A_{1 \jmath}^{1} \longleftrightarrow-\sum A_{j i}^{2}+(n-1) A_{0 j}^{1}-A_{1 \jmath}^{1}=0 \\
& 0=\sum \tilde{T}_{i}^{\frac{l}{j}}=(n-1) A_{1 j}^{0}-A_{1 j}^{0} \longleftrightarrow A_{1 j}^{0}=0 \\
& 0=\Sigma \widetilde{T}_{\bar{i} j}^{\bar{i}}=-\sum A_{j \bar{i}}^{2}+(n-1) A_{1 j}^{1}-A_{1 j}^{1} \longleftrightarrow-\sum A_{j \bar{i}}^{i}+(n-2) A_{1 j}^{1}=0 \\
& 0=\widetilde{T}_{\bar{j} i}^{k}-\widetilde{T}_{\bar{j} k}^{\imath}+\left(\tilde{T}_{\bar{j} \bar{i}}^{\bar{k}}-\tilde{T}_{\bar{j} \bar{k}}^{\bar{i}}\right)= \begin{cases}-4 A_{i j}^{k}-A_{k \bar{i}}^{j}-A_{j \bar{k}}^{i} & (i \neq j \neq k \neq i) \\
-3 A_{i \bar{i}}^{k}-A_{1 k}^{0}-A_{1 \bar{k}}^{1} & (i=j \neq k)\end{cases}
\end{aligned}
$$

From (5.5) and (5.11), we have $-(n-3) A_{0 j}^{0}+(n-1) A_{0 j}^{1}=0$, and so with (5.8), we get $A_{0 j}^{0}=A_{0 j}^{1}=0$, if $n \geq 3$. Then from (5.7) and (5.13), we get $-(n-3) A_{0 j}^{0}-(n-1) A_{1_{j}}^{0}$ $=0$, and so with (5.10), we have $A_{0 j}^{0}=A_{1 j}^{0}=0$, if $n \geq 3$. From the upper formula in (5.6) and $i \leftrightarrow j$ there, we know that $A_{j k}^{2}$ is skew in any two indices, and so again from (5.6) we get $A_{j k}^{2}=0, i \neq j \neq k \neq i$. From the lower formula in (5.6), we get $A_{k i}^{2}=0(i \neq k)$. Similarly from (5.11), we have $A_{j \bar{k}}^{i}=0, i \neq j \neq k \neq i$, and $A_{k i}^{2}=0, i \neq k$.

Now, for (3.5), we have 


$$
\begin{aligned}
& \Psi_{0}^{\prime 0}-\Psi_{0}^{0}=d\left(\chi_{0}^{\prime 0}-\chi_{0}^{0}\right)+\chi^{\prime 0}{ }_{1}^{0} \wedge \chi^{\prime 1}{ }_{0}-\chi_{1}^{0} \wedge \chi_{0}^{1} \\
& =-d\left(A_{01}^{0} \zeta^{1}\right)+\chi^{\prime 0} \wedge \chi^{\prime 1}{ }_{0}^{1}-\left(\chi^{\prime 0}{ }_{1}+A_{11}^{0} \zeta^{1}\right) \wedge\left(\chi^{\prime 1}{ }_{0}+A_{01}^{1} \zeta^{1}\right) \\
& =-d A_{01}^{0} \wedge \zeta^{1}+A_{01}^{0} \sum_{\imath} \zeta^{i} \wedge \zeta^{\bar{i}}-A_{11}^{0} \zeta^{1} \wedge \chi^{\prime 1}-\chi_{0}^{\prime 0}{ }_{1} \wedge A_{01}^{1} \zeta^{1} .
\end{aligned}
$$

Noting that $\chi_{0}^{0}+\chi_{1}^{1}=\chi_{0}^{\prime 0}+\chi^{\prime 1}=0$, we have similarly,

$$
\begin{aligned}
& \Psi_{1}^{\prime 0}-\Psi_{1}^{0}=-d A_{11}^{0} \wedge \zeta^{1}+A_{11}^{0} \sum_{\imath} \zeta^{i} \wedge \zeta^{\bar{i}}-2 A_{01}^{0} \zeta^{1} \wedge \chi_{1}^{\prime 0}-2 A_{11}^{0} \chi_{0}^{\prime 0} \wedge \zeta^{1}, \\
& \Psi_{0}^{\prime 1}-\Psi_{0}^{1}=-d A_{01}^{1} \wedge \zeta^{1}+A_{01}^{1} \sum_{\imath} \zeta^{i} \wedge \zeta^{\bar{i}}+2 A_{01}^{0} \zeta^{1} \wedge \chi_{0}^{\prime 1}+2 A_{01}^{1} \chi_{0}^{\prime 0} \wedge \zeta^{1}, \\
& \Psi_{j}^{\prime}-\Psi_{j}^{i}=-d A_{j 1}^{2} \wedge \zeta^{1}+A_{j 1}^{2} \sum_{\imath} \zeta^{i} \wedge \zeta^{\bar{i}}-A_{j 1}^{k} \chi_{k}^{\prime i} \wedge \zeta^{1}-A_{k 1}^{2} \zeta^{1} \wedge \chi_{j}^{\prime k} .
\end{aligned}
$$

Since we have from (5.3) and (5.4),

$$
\begin{aligned}
& \sum_{i} \widetilde{T}_{1 i}^{i}=-(n-1) A_{01}^{0}, \quad \widetilde{T}_{1 i}-\widetilde{T}_{1 j}^{i}+\left(\tilde{T}_{1 \bar{i}}^{j}-\widetilde{T}_{1 j}^{\bar{i}_{j}}\right)=-2 A_{i 1}^{j}+2 A_{j 1}^{i}=4 A_{j 1}^{i} \quad(i \neq j), \\
& \sum \widetilde{T}_{1 i}^{\bar{i}}=-(n-1) A_{01}^{1}, \quad \sum \widetilde{T}_{1 \bar{i}}^{i}=-(n-1) A_{11}^{0}, \quad \sum \widetilde{T}_{1 \bar{i}}^{\bar{i}_{i}}=-(n-1) A_{11}^{1},
\end{aligned}
$$

(3.5) is written by

$$
\begin{aligned}
& \sum_{i}\left(\widetilde{T}_{1 i}^{i}-\tilde{T}_{0 i \bar{i}}^{0}=-2(n-1) A_{01}^{0},\right. \\
& \sum_{i}\left(\tilde{T}_{1 \bar{i}}^{i}-\tilde{T}_{1 i \bar{i}}^{0}\right)=-2(n-1) A_{11}^{0}, \\
& \sum_{i}\left(\tilde{T}_{1 i}^{\bar{i}}-\tilde{T}_{0 i \bar{i}}^{1}\right)=-2(n-1) A_{01}^{1}, \\
& \tilde{T}_{1 j}^{i}-\tilde{T}_{1 i}^{j}+\tilde{T}_{1 j}^{\bar{i}}-\tilde{T}_{1 \bar{i}}^{j}-\sum_{k} \tilde{T}_{j k \bar{k}}^{i}=-(n+3) A_{j 1}^{i} .
\end{aligned}
$$

Thus we have $A_{01}^{0}=A_{11}^{0}=A_{01}^{1}=A_{j 1}^{2}=0$ and $\chi=\chi^{\prime}$.

Existence: For any Cartan connection $\chi^{\prime}$ of type $H / H_{0}$ on $Q$ such that $\zeta=$ $\chi_{-1}^{\prime}+\chi_{-2}^{\prime}$, we construct a new Cartan connection $\chi^{\prime \prime}$ by setting $\chi_{-1}^{\prime \prime}+\chi_{-2}^{\prime \prime}=\zeta$ and

$$
\chi_{\beta}^{\prime \prime \alpha}=\chi_{\beta}^{\prime \alpha}+A_{\beta \gamma}^{\alpha} \zeta^{\gamma}
$$

where $A_{\beta \gamma}^{\alpha}$ are given by, using the curvature $T^{\prime}$ of $\chi^{\prime}$,

$$
\begin{aligned}
& A_{0 j}^{1}=-\frac{1}{n-2} \Sigma T^{\prime i}{ }_{j i}^{i}, \quad A_{1 j}^{0}=\frac{1}{n-2} \Sigma T^{\prime \prime}{ }_{i j},
\end{aligned}
$$

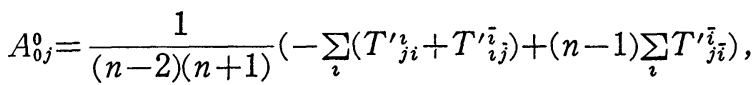

$$
\begin{aligned}
& A_{0 j}^{1}=\sum T^{\prime i}{ }_{j i}-(n-1) A_{0 j}^{0} \text {, } \\
& A_{0 j}^{0}=\frac{1}{(n-2)(n+1)}\left(-\sum_{i}\left(T^{\prime \prime}{ }_{j i}+T^{\prime}{ }_{i j}\right)+(n-1) \sum_{i} T^{\prime 2}{ }_{i j}\right),
\end{aligned}
$$




$$
\begin{aligned}
& A_{1 j}^{0}=(n-1) A_{0 j}^{0}-\Sigma T^{\prime \prime}{ }_{i j} \text {. }
\end{aligned}
$$

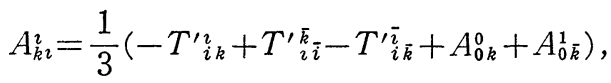

$$
\begin{aligned}
& A_{i \bar{i}}^{k}=\frac{1}{3}\left\{T_{i \bar{i}}^{k_{i}}-T_{k \bar{i}}^{\prime \prime}-T_{\bar{k} \bar{i}}^{\prime \bar{i}}+A_{1 k}^{0}+A_{0 \bar{k}}^{0}\right\} .
\end{aligned}
$$

To obtain $A_{j k}^{2}$, take a cyclic sum of the upper formula in (5.9), noting that $T_{j \imath}^{k}-T_{j k}^{\imath}+T_{j i}^{\bar{k}_{i}}-T_{j \bar{k}}^{\bar{i}}=0$,

$$
\Im_{i, j, k} S_{i j}^{k}=-6 S,
$$

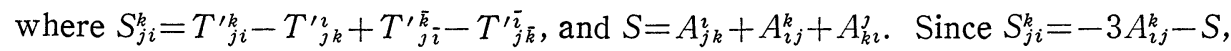
we get

$$
A_{\imath \jmath}^{k}=-\frac{1}{3}\left(S_{j i}^{k}-\frac{1}{6} \Im_{i, \jmath, k} S_{j i}^{k}\right) .
$$

Similarly from (5.14), we get

$$
A_{i j}^{k}=-\frac{1}{3}\left(S_{j i}^{k}-\frac{1}{6} \Im_{i, j, k} S_{j i}^{k}\right) .
$$

Let $\phi^{\prime \prime}=(1 / 2) T^{\prime \prime} \zeta \wedge \zeta$ be the curvature form of $\chi^{\prime \prime}$. Define a connection $\chi$ by $\chi_{-2}=\chi_{-2}^{\prime \prime}, \chi_{-1}=\chi_{-1}^{\prime \prime}$ and

$$
\chi_{\beta}^{\alpha}=\chi_{\beta}^{\prime \prime}{ }_{\beta}^{\alpha}+A_{\beta 1}^{\alpha} \zeta^{1},
$$

where $A_{\beta 1}^{\alpha}$ is given by $(5.15) \sim(5.18)$, in which $\tilde{T}$ is replaced by $T^{\prime \prime}$. Then we can see easily that the obtained $\chi$ satisfies $T^{-1}=\partial^{*} T^{0}=\partial^{*} T^{1}\left(e_{1}\right)=0$. Since there always exists a Cartan connection $\chi^{\prime}$ of type $H / H_{0}$ with basic form $\zeta$ by the local triviality of the bundle, we get $\chi$ actually by above procedure. q.e.d.

Proof of Proposition 5.3. Let $(Q, \zeta)$ be an $H_{0}$-reduction of $(P, \theta)$ satisfying

$$
d \zeta_{-2}+\frac{1}{2}\left[\zeta_{-1}, \zeta_{-1}\right]=0 \text {, }
$$

and let $\chi$ be the Cartan connection of type $H / H_{0}$ constructed in Proposition 5.2. Now, let $\omega^{\prime}$ be a connection on $P$ naturally i. e. flattly extended from $\chi$. Then $\omega^{\prime}$ restricted to $Q$ satisfies

$$
\left(\omega^{\prime 1}, \omega^{\prime 2}, \omega^{\prime i}, \omega_{\beta}^{\prime \alpha}\right)=\left(\zeta^{1}, \zeta^{i}, \zeta^{i}, \chi_{\beta}^{\alpha}\right),
$$

where $i=1, \cdots, n,(\alpha, \beta)=(0,0),(0,1),(1,0),(i, j), 2 \leqq i \neq j \leqq n$. Using this $\omega^{\prime}$, we construct a unique normal Cartan connection $\omega$ of type $G / G^{\prime}$ such that

$$
\left(\omega^{1}, \omega^{\imath}, \omega^{i}, \omega_{\beta}^{\alpha}\right)=\left(\omega^{\prime 1}, \omega^{\prime 2}, \omega^{\prime i}, \omega_{\beta}^{\prime \alpha}\right),
$$

where $(\alpha, \beta)=(0,0),(0,1),(1,0),(i, j), 2 \leqq i \neq j \leqq n$. We denote the basic form on $P$ by $\theta=\omega_{-2}^{\prime}+\omega_{-1}^{\prime}=\omega_{-2}+\omega_{-1}, \iota^{*} \theta=\zeta$. 
Uniqueness: Let $\omega$ and $\omega^{\prime}$ be two normal Cartan connections of type $G / G^{\prime}$, satisfying (5.20). Since $\omega-\omega^{\prime}=0$ on each fibre and by (5.20). We can write

Now, from

$$
\begin{aligned}
& \omega_{1}^{1}-\omega_{1}^{\prime 1}=A_{1 \gamma}^{1} \theta^{\gamma}, \\
& \omega_{i}-\omega_{i}^{\prime}=A_{i \gamma} \theta^{\gamma}, \\
& \omega_{\bar{i}}-\omega_{\bar{i}}=A_{\bar{i} \gamma} \theta^{\gamma}, \\
& \omega_{1}-\omega_{1}^{\prime}=A_{1 \gamma} \theta^{\gamma} .
\end{aligned}
$$

$$
\Omega^{\prime 1-} \Omega^{1}=-A_{1 \gamma}^{1} \theta^{r} \wedge \theta^{1},
$$

we get $A_{1 j}^{1}=A_{1 j}^{1}=0,2 \leqq j \leqq n$. Then from

$$
\begin{aligned}
& \Omega^{\prime \imath}-\Omega^{\imath}=A_{\bar{\imath} \gamma} \theta^{r} \wedge \theta^{1}, \\
& \Omega^{\prime \bar{\imath}}-\Omega^{\bar{i}}=-A_{\imath \gamma} \theta^{r} \wedge \theta^{1}+\theta^{\bar{\imath}} \wedge A_{11}^{1} \theta^{1},
\end{aligned}
$$

we have, denoting $\tilde{K}=K^{\prime}-K, \tilde{K}_{1 \jmath}^{i}=-A_{i \jmath}, \tilde{K}_{1 \jmath}^{\bar{i}}=A_{\imath \jmath}, \tilde{K}_{1 j}^{\imath}=-A_{i j}, \tilde{K}_{1 j}^{i}=A_{\imath j}-A_{11}^{1} \delta_{\jmath}^{\imath}$, and other $\tilde{K}_{\beta \gamma}^{\alpha}=0$. From

$$
\begin{aligned}
& \Omega_{0}^{\prime 0}-\Omega_{1}^{1}=-\theta^{2} \wedge A_{\imath \gamma} \theta^{r}-\theta^{1} \wedge A_{1 \gamma} \theta^{r}, \\
& \Omega_{1}^{\prime 0}-\Omega_{1}^{0}=-\theta^{2} \wedge A_{i \gamma} \theta^{r}, \\
& \Omega_{0}^{\prime 1}-\Omega_{0}^{1}=-\theta^{i} \wedge A_{\imath \gamma} \theta^{r}, \\
& \Omega_{1}^{\prime 1}-\Omega_{1}^{1}=-d\left(A_{11}^{1} \theta^{1}\right)-\theta^{i} \wedge A_{i \gamma} \theta^{r}-\theta^{1} \wedge A_{1 \gamma} \theta^{r}, \\
& \Omega^{\prime \prime}{ }_{j}-\Omega_{j}^{i}=-A_{\imath \gamma} \theta^{r} \wedge \theta^{\jmath}-A_{i \gamma} \theta^{r} \wedge \theta^{j}-\theta^{\imath} \wedge A_{\jmath \gamma} \theta^{r}-\theta^{\bar{\imath}} \wedge A_{j \gamma} \theta^{r},
\end{aligned}
$$

we have by (3.5),

(1) $0=\sum_{\imath}\left(\tilde{K}_{1 i}^{\imath}-K_{0 i \bar{\imath}}^{0}\right)=\sum_{\imath}\left(-A_{i \imath}+A_{i \bar{i}}\right)$,

(2) $0=\sum_{\imath}\left(\tilde{K}_{1 \bar{i}}^{\imath}-\tilde{K}_{1 i \bar{\imath}}^{0}\right)=-\sum_{\imath}\left(-A_{\bar{i} \bar{\imath}}+A_{\bar{\imath} \bar{i}}\right)$,

(3) $0=\sum_{\imath}\left(\tilde{K}_{1 i}^{\bar{i}}-\tilde{K}_{0 i i}^{1}\right)=\sum_{\imath}\left(A_{i i}-A_{i i}\right)$,

(4) $0=\sum_{\imath}\left(\tilde{K}_{1 \bar{i}}^{i_{i}}-\tilde{K}_{1 i \bar{i}}^{1}\right)=-2(n-1) A_{11}^{1}+\sum_{\imath}\left(A_{\imath i}-A_{\bar{\imath} \imath}\right)$,

(5) $0=\tilde{K}_{1 j}^{i}-\tilde{K}_{1 i}^{j}+\tilde{K}_{1 j}^{\bar{i}}-\tilde{K}_{1 \bar{i}}^{j}-\sum_{k} \tilde{K}_{j k \bar{k}}^{i}=-A_{i j}+A_{j i}+A_{\imath j}-A_{j \bar{i}}-A_{\imath j}+A_{i j}+A_{j \bar{i}}-A_{j i}$.

From (1) and (4), we get $A_{11}^{1}=0$. Note that (2), (3), (5) hold trivially. Then (3.6) is written from $\tilde{K}_{1 j}^{i}=A_{\imath \jmath}, \tilde{K}_{0 \imath \jmath}^{0}=-A_{\imath j}+A_{j i}, \tilde{K}_{0 i \jmath}^{1}=-A_{\imath \jmath}, \tilde{K}_{m k \jmath}^{i}=-A_{i k} \delta_{m}^{\jmath}+$ $A_{\imath j} \delta_{k}^{m}-A_{m j} \delta_{k}^{2}+A_{m k} \delta_{\jmath}^{i}$, 


$$
0=A_{j i}-n A_{\imath j}-\sum_{k} A_{k k} \delta_{j}^{\imath},
$$

from $\tilde{K}_{1 \jmath}^{\imath}=-A_{i \jmath}, \tilde{K}_{1 \imath \jmath}^{0}=-A_{\bar{i} j}+A_{j i}, \tilde{K}_{1 \bar{\imath} \jmath}^{1}=-A_{\bar{\imath} \jmath}, \tilde{K}_{m \bar{k} \jmath}^{i}=-A_{i \bar{k}} \delta_{m}^{\jmath}+A_{\bar{i} j} \delta_{m}^{k}+A_{m \bar{k}} \delta_{\jmath}^{i}$ $-A_{\bar{m} j} \delta_{k}^{2}$,

$$
0=-(n+1) A_{\bar{i} j}+A_{i \bar{j}}-\sum A_{k \bar{k}} \delta_{j}^{2}+A_{j i},
$$

from $\tilde{K}_{1 j}^{\bar{i}}=A_{\imath j}, \quad \tilde{K}_{0 \imath j}^{0}=-A_{\imath j}, \quad \tilde{K}_{0 \bar{j} j}^{1}=-A_{\imath j}+A_{j \bar{i}}, \quad \tilde{K}_{m k j}^{i}=A_{\imath j} \delta_{k}^{m}-A_{\bar{i} k} \delta_{m}^{j}-A_{m j} \delta_{k}^{i}+$ $A_{\bar{m} k} \delta_{\jmath}^{\imath}$.

$$
0=-(n+1) A_{i j}+A_{\bar{i}_{j}}-\sum A_{\bar{k} k} \delta_{j}^{i}+A_{j \bar{i}},
$$

and from $\tilde{K}_{1 j}^{i}=-A_{i \bar{j}}, \quad \tilde{K}_{1 \imath j}^{0}=-A_{\bar{i} j}, \quad \tilde{K}_{1 i \bar{j}}^{1}=-A_{\bar{i} j}+A_{j \bar{i}}, \quad \tilde{K}_{m \bar{k} j}^{i}=-A_{\bar{i} \bar{k}} \delta_{m}^{\jmath}+A_{i j} \delta_{k}^{m}-$

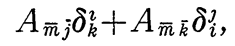

$$
0=A_{\bar{j} \bar{i}}-n A_{\bar{i} \dot{j}}-\sum A_{\bar{k} \bar{k}} \delta_{j}^{2} .
$$

By (6) and $\imath \leftrightarrow j$ in (6), when $i \neq j$, we get $A_{\imath \jmath}=0$. Similarly from (9), we have $A_{i \bar{j}}=0 \quad i \neq j$. Putting $\imath=j$ in (6) and in (7), we get $A_{i i}=A_{\bar{i} \bar{i}}=0$. Now, from (7), (8) and $\imath \leftrightarrow j$ in (7), (8), we get $A_{i \jmath}=A_{\imath j}=0(i \neq j)$. Putting $i=j$ in (7) and in (8), we get $A_{i \bar{i}}=A_{\bar{\imath} \imath}=0$. Note that (1) and (4) are satisfied. Therefore we can write

and

$$
\omega_{i}-\omega_{\imath}^{\prime}=A_{i 1} \theta^{1}, \quad \omega_{\bar{i}}-\omega_{\bar{i}}^{\prime}=A_{\bar{i} 1} \theta^{1}, \quad \text { and } \quad \omega_{1}-\omega_{1}^{\prime}=A_{1 \gamma} \theta^{r}
$$

$$
\begin{aligned}
& \Omega_{0}^{\prime 0}-\Omega_{0}^{0}=-\theta^{2} \wedge A_{i 1} \theta^{1}-\theta^{1} \wedge A_{1 \gamma} \theta^{\gamma}, \quad \Omega_{1}^{\prime 0}-\Omega_{1}^{0}=-\theta^{2} \wedge A_{\bar{i} 1} \theta^{1}, \\
& \Omega_{0}^{\prime 1}-\Omega_{0}^{1}=-\theta^{\bar{i}} \wedge A_{\imath 1} \theta^{1}, \quad \Omega_{1}^{\prime 1}-\Omega_{1}^{1}=-\theta^{\bar{i}} \wedge A_{\bar{i} 1} \theta^{1}-\theta^{1} \wedge A_{1 \gamma} \theta^{r}, \\
& \Omega^{\prime}{ }_{j}-\Omega_{j}{ }_{j}=-A_{i 1} \theta^{1} \wedge \theta^{\jmath}-A_{\bar{i} 1} \theta^{1} \wedge \theta^{\bar{i}}-\theta^{2} \wedge A_{j 1} \theta^{1}-\theta^{\bar{i}} \wedge A_{j 1} \theta^{1}, \\
& \Omega^{\prime}{ }_{i}-\Omega_{i}=-d\left(A_{i 1} \theta^{1}\right)-A_{i 1} \theta^{1} \wedge \omega_{0}^{0}-A_{\bar{i} 1} \theta^{1} \wedge \omega_{0}^{1}+\omega_{j}^{2} \wedge A_{j 1} \theta^{1}-\theta^{\bar{i}} \wedge A_{1 \gamma} \theta^{r}, \\
& \Omega^{\prime}{ }_{i}-\Omega_{i}=-d\left(A_{\bar{i} 1} \theta^{1}\right)-A_{i 1} \theta^{1} \wedge \omega_{1}^{0}-A_{\bar{i} 1} \theta^{2} \wedge \omega_{1}^{1}+\omega_{i}^{\jmath} \wedge A_{j 1} \theta^{1}+\theta^{2} \wedge A_{1 \gamma} \theta^{r} .
\end{aligned}
$$

Noting that $d \theta^{1} \equiv-\Sigma \theta^{2} \wedge \theta^{i}\left(\bmod \theta^{1}\right)$, we have

$$
\tilde{K}_{i j \bar{k}}=A_{i 1} \delta_{j}^{k}+A_{1 j} \delta_{i}^{k}, \quad \tilde{K}_{\bar{i} j \bar{k}}=A_{\bar{i} 1} \delta_{j}^{k}+A_{1 \bar{k}} \delta_{i}^{\jmath} .
$$

Thus togather with

$$
\tilde{K}_{0 i 1}^{0}=-A_{\imath 1}+A_{1 \imath}, \quad \tilde{K}_{0 i \overline{1}}^{1}=-A_{i 1}, \quad \tilde{K}_{j k 1}^{\imath}=A_{\imath 1} \delta_{k}^{\jmath}-A_{j 1} \delta_{k}^{\imath},
$$

we obtain for (3.7)

$$
0=-(2 n-1) A_{i 1} .
$$

Similarly from $\tilde{K}_{1 i 1}^{0}=-A_{\bar{i} 1}, \tilde{K}_{1 \bar{i} 1}^{1}=-A_{\bar{i} 1}+A_{1 \bar{i}}, \tilde{K}_{j \bar{k} 1}^{i_{1}}=A_{\bar{i} 1} \delta_{k}^{j}-A_{j_{1}} \delta_{k}^{2}$, it follows

$$
0=-(2 n-1) A_{\bar{i}_{1}} \text {. }
$$


On other hand, for (3.8), we have from $\tilde{K}_{01 \jmath}^{0}=A_{j 1}-A_{1 \jmath}, K_{11}^{1}=-A_{1 \jmath}, K_{i j k}=$ $A_{1 k} \delta_{j}^{i}-A_{1 j} \delta_{k}^{i}, K_{\imath j k}=-A_{i 1} \delta_{k}^{j}-A_{1 k} \delta_{j}^{i}$

$$
0=-(2 n-1) A_{1 \jmath},
$$

and from $K_{01 j}^{0}=-A_{1 j}, K_{11 j}^{1}=A_{j 1}-A_{1 j}, K_{\bar{i} k j}=A_{\bar{i} 1} \delta_{k}^{\jmath}+A_{1 j} \delta_{k}^{2}, K_{i \bar{k} j}=-A_{1 j} \delta_{k}^{2}+A_{1 \bar{k}} \delta_{j}^{2}$,

$$
0=-(2 n-1) A_{1 j} \text {. }
$$

Finally, from

$$
\Omega_{1}^{\prime}-\Omega_{1}=-d\left(A_{11} \theta^{1}\right)+\left(\omega_{0}^{0}+\omega_{1}^{1}\right) \wedge A_{11} \theta^{1},
$$

we get $K_{1 i \bar{i}}=A_{11}$ and (3.9) is given by

$$
0=-3(n-1) A_{11}
$$

Thus we obtain $\omega^{\prime}=\omega$.

Existence: Let $\omega^{\prime}$ be any Cartan connection of type $G / G$ on $P$, satisfying (5.19). We make the desired normal connection $\omega$ satisfying (5.20) by

$$
\omega_{1}^{1}=\omega_{1}^{\prime 1}+A_{1 \gamma}^{1} \theta^{r}, \quad \omega_{i}=\omega_{i}^{\prime}+A_{\imath \gamma} \theta^{\gamma}, \quad \omega_{\bar{\imath}}=\omega_{\bar{i}}^{\prime}+A_{\bar{\imath} \gamma} \theta^{r}, \quad \omega_{1}=\omega_{1}^{\prime}+A_{1 \gamma} \theta^{r},
$$

where $A_{1 \gamma}^{1}, A_{\imath \gamma}$ and $A_{i \gamma}$ are chosen as follows: Since we put $\tilde{K}=K^{\prime}-K$ in above calculation, in order that the new $\omega$ is normal, we have for (3.5).

(1) $\sum_{i}\left(K_{1 i}^{\prime 2}-K_{0 i i}^{\prime 0}\right)=\sum_{i}\left(-A_{i i}+A_{i \bar{i}}\right)$,

(4) $\sum_{\imath}\left(K_{1 i \bar{i}}^{\prime \bar{i}}-K^{\prime 1}{ }_{1 i \bar{i}}\right)=-2(n-1) A_{11}^{1}+\sum_{\imath}\left(A_{i \bar{i}}-A_{\bar{\imath} \imath}\right)$,

for (3.6),

$(6)^{\prime}-K_{1 j}^{\prime \bar{i}}+K^{\prime 0}{ }_{02 j}+K^{\prime 1}{ }_{0 i j}-\sum K_{k k j}^{\prime 2}=A_{j i}-n A_{\imath j}-\sum A_{k k} \delta_{j}^{\imath}$,

(7) $\quad K^{\prime \prime}{ }_{1 j}+K^{\prime 0}{ }_{1 i j}+K^{\prime 1}{ }_{1 i} j-\sum K^{\prime 2}{ }_{k \bar{k} j}=-(n+1) A_{\bar{i} j}+A_{\imath \bar{j}}+A_{\bar{j} i}+A_{11}^{1} \delta_{j}^{i}-\sum A_{k \bar{k}} \delta_{j}^{\imath}$,

$(8)^{\prime}-K^{\prime{ }_{1 j}}+K^{\prime 0}{ }_{02 j}+K^{\prime 1}{ }_{0 i \bar{j}}-\sum K_{k k}^{\prime \imath}=-(n+1) A_{2 j}+A_{\bar{i} j}+A_{j \bar{i}}-A_{11}^{1} \delta_{j}^{i}-\sum A_{\bar{k} k} \delta_{j}^{i}$,

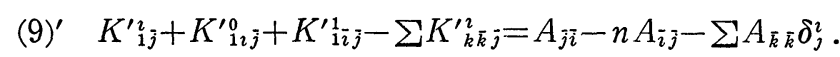

From $(6)^{\prime}$, and $i \leftrightarrow j$ in $(6)^{\prime}$ where $i \neq j$, we get

$$
A_{\imath \jmath}=\frac{1}{1-n^{2}}\left\{n\left(-K^{\prime i}{ }_{1 j}+K^{\prime 0}{ }_{02 j}+K^{\prime 1}{ }_{0 i j}-\sum_{k} K_{k k j}^{\prime 2}\right)-K^{\prime}{ }_{1 i}+K^{\prime}{ }_{0 j i}+K^{\prime 1}{ }_{0 j i}-\sum_{k} K_{k k i}^{\prime}\right\} \text {. }
$$

Summing over $i=j$ in $(6)^{\prime}$, we get

$$
-2(n-1) \sum_{\imath} A_{i i}=\sum_{\imath}\left(-K^{\prime \bar{i}}{ }_{1 i}+K^{\prime 1}{ }_{0 i i}-\sum_{i, k} K_{k k \imath}^{i}\right),
$$

thus put 


$$
A_{2 i}=\frac{1}{(1-n)}\left\{-K^{\prime{ }_{1 i}}+K^{\prime 1}{ }_{0 i}-\sum K_{k k i}^{i}+\frac{1}{2(1-n)} \sum_{i}\left(-K^{\prime{ }_{1 i}}+K^{\prime 1}{ }_{0 i}-\sum_{k} K_{k k i}^{\prime \imath}\right)\right\} .
$$

Similarly from (9), we obtain

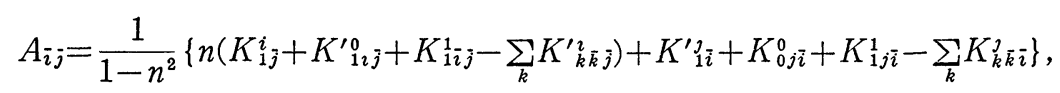

for $i \neq j$, and

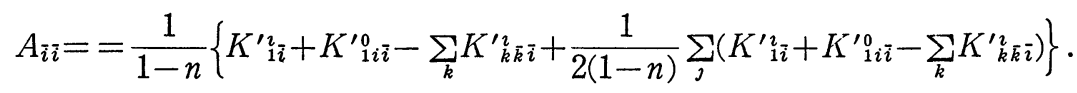

Now, when $i \neq j,(7)^{\prime},(8)^{\prime}, i \leftrightarrow j$ in $(7)^{\prime},(8)^{\prime}$ are the equations among four variables $A_{\bar{\imath} \jmath}, A_{j i}, A_{\imath j}, A_{j i}$. Denoting by $L_{\imath \jmath}$ and $M_{\imath \jmath}$ the left hand sides of (7)' anp $(8)^{\prime}$, respectively, we solve the equations and get

$$
\begin{aligned}
& A_{i \jmath}=\frac{1}{4-(n+1)^{2}}\left\{M_{\imath j}-\frac{2-(n+1)^{2}}{n+1} L_{\imath j}+\frac{2}{n+1} M_{j i}+L_{j i}\right\}, \\
& A_{\imath j}=\frac{1}{4-(n+1)^{2}}\left\{L_{\imath j}-\frac{2-(n+1)^{2}}{n+1} M_{\imath j}+\frac{2}{n+1} L_{j i}+M_{j i}\right\} .
\end{aligned}
$$

When $i=j$, from $(1)^{\prime}$ and $(4)^{\prime}$, we get

$$
A_{11}^{1}=\frac{1}{2(n-1)} \sum_{i}\left(K_{1 i}^{\prime 2}-K^{\prime 0}{ }_{0 i \bar{i}}-K^{\prime i}{ }_{1 i}+K_{1 i \bar{i}}^{1}\right) .
$$

Now, putting $S=\sum A_{k \bar{k}}$ and $T=\sum A_{\bar{k} k}$, we obtain

$$
\sum\left(L_{i i}+M_{i i}\right)=-2(n-1)(S+T) .
$$

Since $S-T$ is given by $(1)^{\prime}$, we have

$$
\begin{aligned}
& S=\frac{1}{2} \sum_{\imath}\left(-\frac{1}{2(n-1)}\left(L_{i i}+M_{i i}\right)+K^{\prime i}{ }_{1 i}-K^{\prime 0 i i}\right\} \text {, } \\
& T=\frac{1}{2} \Sigma\left(-\frac{1}{2(n-1)}\left(L_{i i}+M_{i i}\right)-K^{\prime i}{ }_{1 i}+K^{\prime 0}{ }_{0 i i}\right\} \text {. }
\end{aligned}
$$

Finally from $(7)^{\prime}$ and $(8)^{\prime}$,

$$
n L_{i i}+M_{i i}=\left(1-n^{2}\right) A_{\bar{i} i}-n S-T+(n-1) A_{11}^{1},
$$

and we get

$$
A_{\bar{\imath} \imath}=\frac{1}{1-n^{2}}\left(n L_{i i}+M_{i i}+n S+T-(n-1) A_{11}^{1}\right),
$$

and similarly,

$$
A_{i \bar{i}}=\frac{1}{1-n^{2}}\left(n M_{i i}+L_{i i}+n T+S+(n-1) A_{11}^{1}\right) .
$$


Now, let $\omega^{\prime \prime}$ be a connection on $(P, \theta)$ such that $\left(\omega^{\prime \prime 1}, \omega^{\prime \prime \imath}, \omega^{\prime \prime}, \omega_{\beta}^{\prime \prime}\right)=$ $\left(\omega^{\prime 1}, \omega^{\prime 2}, \omega^{\prime i}, \omega_{\beta}^{\prime \alpha}\right),(\alpha, \beta)$ as in $(5.20)$, and

$$
\begin{aligned}
& \omega^{\prime \prime}{ }_{1}^{1}=\omega_{1}^{\prime 1}+A_{11}^{1} \theta^{1}, \omega^{\prime \prime}{ }_{i}=\omega_{i}^{\prime}+A_{\imath j} \theta^{j}+A_{i j} \theta^{j}, \\
& \omega^{\prime \prime}{ }_{i}=\omega_{i}^{\prime}+A_{i j} \theta^{j}+A_{\bar{i} j} \theta^{j}, \omega_{1}^{\prime \prime}=\omega^{\prime}{ }_{1},
\end{aligned}
$$

where $A_{11}^{1}, A_{\imath \jmath}, A_{\imath \bar{\jmath}}, A_{\bar{\imath} \jmath}, A_{\bar{\imath}}$ are obtained above, using the curvature of $\omega^{\prime}$ and $A$. Then $\hat{\omega}$ given by

$$
\widehat{\omega}_{i}=\omega^{\prime \prime}{ }_{i}+A_{i 1} \theta^{1}, \quad \hat{\omega}_{i}=\omega^{\prime \prime}{ }_{i}+A_{i 1} \theta^{1}, \quad \hat{\omega}_{1}=\omega^{\prime}{ }_{1}+A_{1 j} \theta^{j}+A_{1 j} \theta^{j}
$$

satisfies $\hat{K}^{-1}=\partial^{*} \hat{K}^{0}=\partial^{*} \hat{K}^{1}=0$, where $\hat{K}$ is the curvature of $\hat{\omega}$. Moreover, it satisfies $\left(\partial^{*} \hat{K}^{2}\right)\left(e_{1}\right)=0$, if

$$
\begin{aligned}
& K^{\prime \prime}{ }_{0 i 1}^{0}+K^{\prime \prime}{ }_{0 i \overline{1} 1}-\sum_{k}\left(K^{\prime \prime \prime}{ }_{k k 1}+K^{\prime \prime}{ }_{i k \bar{k}}\right)=-(2 n-1) A_{i 1}, \\
& K^{\prime \prime}{ }_{1 i 1}+K^{\prime \prime}{ }_{1 \bar{i} 1}-\sum_{k}\left(K^{\prime \prime l_{\bar{k} 1}}+K^{\prime \prime}{ }_{i k \bar{k}}\right)=-(2 n-1) A_{\bar{i}_{1}},
\end{aligned}
$$

and $\left(\partial^{*} \hat{K}^{2}\right)\left(g_{-1}\right)=0$, if

$$
\begin{aligned}
& \left(K^{\prime \prime 0}{ }_{01 j}+K^{\prime \prime 1}{ }_{11 j}\right)-\sum_{\imath}\left(K^{\prime \prime}{ }_{i \imath j}-K^{\prime \prime}{ }_{i \bar{\jmath}}\right)=-(2 n-1) A_{1 \jmath}, \\
& \left(K^{\prime \prime 0}{ }_{01 j}^{0}+K^{\prime \prime 1}{ }_{11 j}\right)-\sum_{\imath}\left(K^{\prime \prime}{ }_{i \imath j}-K^{\prime \prime}{ }_{i \bar{j} j}\right)=-(2 n-1) A_{1 j} .
\end{aligned}
$$

Determine $\mathrm{A}_{i 1}, A_{\bar{i} 1}, A_{1 j}$ and $A_{1 j}$ by these formulas. Finally, let $\omega$ be such that $\omega=\widehat{\omega}$ except for

$$
\omega_{1}=\widehat{\omega}_{1}+A_{11} \theta^{1},
$$

where $A_{11}$ is given by, using the curvature $\hat{K}$ of $\hat{\omega}$,

$$
\sum_{i}\left(-\hat{K}_{i i 1}+\hat{K}_{i \bar{i} 1}-\hat{K}_{1 i \bar{i}}\right)=-3(n-1) A_{11} .
$$

Since

$$
\begin{aligned}
& \hat{\Omega}_{i}-\Omega^{\prime \prime}{ }_{i}=d\left(A_{i 1} \theta^{1}\right)+A_{1 j} \theta^{i} \wedge \theta^{j}, \\
& \hat{\Omega}_{i}-\Omega^{\prime \prime}{ }_{i}=-A_{1 j} \theta^{i} \wedge \theta^{j}, \\
& \hat{\Omega}_{1}-\Omega^{\prime \prime}{ }_{1}=d\left(A_{1 i} \theta^{i}\right),
\end{aligned}
$$

we get

$$
\sum_{i}\left(-K^{\prime \prime}{ }_{i i 1}+K^{\prime \prime}{ }_{i \bar{i} 1}+\partial_{\bar{i}} A_{i 1}-K^{\prime \prime}{ }_{1 i \bar{i}}+\partial_{\bar{i}} A_{12}\right)=-3(n-1) A_{11} .
$$

Then by above construction, starting from $\omega^{\prime}$ satisfying (5.19) which exists by the local triviality of the bundle, we get a normal Cartan connection $\omega$ of type $G / G^{\prime}$ on $P$.

q.e.d. 


\section{REFERENCES}

[B] Blair, D., Contact manifolds in Riemannian geometry, Lecture Note in Math. 509, Springer (1976).

[C C ] Cecil, T. And Chern, S.S., Tautness and Lie sphere geometry, Math. Ann. 278 (1987), 381-399.

[K] Kostant, B., Lie algebra cohomology and the generalized Borel-Weil theorem, Ann. of Math., 74 (1961), 329-387.

[KN] Kobayashi, S. And Nagano, T., On projective connections, J. of Math. and Mech., 13 (1964), 215-235.

[KNI] Kobayashi, S. and Nomizu, K., Foundations of Differential geometry I, Interscience Pub., New York-London (1963).

[M1] MiYaokA, R., Dupin hypersurfaces and a Lie invariant, Kodai Math. J., 12 (1989), 228-256.

[M2] MiYAoKA, R., Lie contact structures and conformal structures, to appear in Kodai Math. J.

[M3] MiyaokA, R., A note on Lie contact manifolds, Preprint, Tokyo Institute of Technology (1990).

[O] OGiue, K., Theory of conformal connections, Kodai Math. Sem. Rep. 19 (1967), 193-224.

[P] Pinkall, U., Dupin hypersurfaces, Math. Ann., 270 (1985), 427-440.

[S1] SASAKI, S., On the differential geometry of tangent bundles of Riemannian manifolds, Tohoku Math. J., 10 (1958), 338-354.

[S2] SASAKI, S., On differential manifolds with certrain structures closely related to almost contact structures I, Tohoku Math. J., 1 (1960), 459-476.

[S3] SASAKI, S., On the differential geometry of tangent bundles of Rimannian manifolds II, Tohoku Math. J., 14 (1962), 146-155.

[S] SATo, H., Lie's contact geometry and Lie manifolds (in Japanese) Proc. of 35th Top Symp. (1987), Ryukyu Univ., 41-63.

[S Y] Sato, H. AND YamaGuchi, K., Lie contact manifolds, "Geometry of Manifolds (edited by K. Shiohama)", Acad. Press, Boston (1989), 191-238.

[T1] TANAKA, N., On differential systems, graded Lie algebras and pseudo groups, J. Math. of Kyoto Univ. 10 (1970), 1-82.

[T2] TANAKA, N., On non-degenerate real hypersurfaces, graded Lie algebras and Cartan connections, Japan J. Math., 2 (1976), 131-190.

[T3] TANAKA, N., On the equivalence problems associated with simple graded Lie algebras, Hokkaido Math. J., 8 (1979), 23-84.

Department of Mathematics

TOKyo Institute of TECHNOLOGY

Ohokayama, Meguroku, 152

TOKYO/JAPAN 